\title{
Optical methods for measuring corneal topography: A review
}

\section{Métodos ópticos para medición de topografía corneal: Una revisión}

\author{
Jaime Misael Jalife-Chavira *, Gerardo Trujillo-Schiaffino, Paloma Guadalupe Mendoza- \\ Villegas, Didia Patricia Salas-Peimbert, Marcelino Anguiano-Morales \\ Luis Francisco Corral-Martínez.
}

Tecnológico Nacional de México/ I.T. Chihuahua, Ave. Tecnológico 2909, 31310 Chihuahua, México.

${ }^{(*)}$ E-mail: jalife.misael@gmail.com

Received: 30/11/2018 Accepted: $16 / 04 / 2019$

DOI: $10.7149 /$ OPA.52.2.51016

\begin{abstract}
:
The cornea is the outermost surface of the eyeball; and has almost $70 \%$ of the eyes total refractive power, what makes it essential for the human vision process. However, some pathologies and refractive errors modify normal functions of the cornea, decreasing visual acuity along with the individual's quality of life. Thus, in the presence of ametropia, an appropriate diagnosis, treatment, and corrections of cornea's dimensions is vital. Nowadays, several methods to retrieve corneal topography are available. In this paper, the most relevant techniques in the field are presented.
\end{abstract}

Key words: cornea, topography, curvature radius

\section{RESUMEN:}

La córnea es la superficie más externa del globo ocular, siendo responsable de al menos 70\% del poder total refractivo del ojo, lo que la convierte en esencial para el proceso de la visión humana. Sin embargo, su funcionamiento puede ser modificado por diferentes patologías y errores refractivos, provocando la disminución de la agudeza visual a la par de la calidad de vida del individuo. Por lo tanto, en presencia de alguna ametropía es vital un apropiado diagnóstico, tratamiento y corrección de las dimensiones de la córnea. Hoy en día existen varios métodos para adquirir la topografía corneal. En este artículo se presentan las técnicas más relevantes en el área.

Palabras clave: córnea, topografía, radio de curvatura

\section{REFERENCES AND LINKS / REFERENCIAS Y ENLACES}

[1] L. Remington, Clinical Anatomy and Physiology of the Visual System. St. Louis MO, Elsevier (2012).

[2] M. Sridhar, "Anatomy of cornea and ocular surface," Indian Journal of Ophthalmology 66, 190-194 (2018).

[3] Herbert Gross, Handbook of Optical Systems: Vol. 4 Survey of Optical Instruments. Weinheim Germany, Wiley-Vch Verlag GmbH \& Co (2008).

[4] W. Ansari, A. Nadeem, Atlas of Ocular Anatomy. Switzerland, Springer (2016).

[5] S. Dua, A. Faraj, G. Said, T. Gray, J. Lowe, "Human Corneal Anatomy Redefined A Novel PreDescemet's Layer (Dua's Layer)," Ophthalmology 120, 1778-1785 (2013).

[6] D. Schanzlin, J. Robin, Corneal Topography: Measuring and Modifying the Cornea. New York USA, Springer Science \& Business Media (2012).

[7] A. Calossi, "Corneal Asphericity and Spherical Aberration," Journal of Refractive Surgery 23, 505514 (2007).

[8] G. Smith, D. Atchison, The eye and visual optical instruments. Cambridge UK, Cambridge University Press (1997). 
[9] P. Artal, Handbook of Visual Optics Fundamentals and Eye Optics Vol. 1. Boca Raton FL, USA, CRC PressTaylor \& Francis Group (2017).

[10] S. Rojas, A. Saucedo, Oftalmología. México, Manual Moderno (2014).

[11] S. Boyd, Distrofias y Degeneraciones Corneales. Rep. De Panamá, JP Medical (2012).

[12] M. Yanoff, J. Duker, Ophthalmology. St Louis US, Elsevier Health Sciences (2013).

[13] F. Boyd, Abberrometers and Corneal Topography. Rep. De Panamá Highlights of Ophthalmology International (2003).

[14] A. Agarwal, A. Agarwal, S. Jacob, Dr Agarwal's Textbook on Corneal Topography Including Pentacam and Anterior Segment OCT. New Delhi, India, Jaypee Brothers Medical Publishers (2010).

[15] M. Sinjab, Corneal Topography in Clinical Practice (Pentacam System) Basics and Clinical Interpretation, New Delhi, India, Jaypee Brothers Medical Publishers (2009).

[16] M. Wang, L. Kugler, Atlas and Clinical Reference Guide for Corneal Topography. Thorofare, NJ, USA, SLACK Incorporated (2014).

[17] S. Agarwal, A. Agarwal, Topografía corneal: paso a paso. México, Santiago Auroch (2011).

[18] Oculus Pentacam®, "Interpretation Guide 3rd edition" (OCULUS Optikgeräte GmbH 2014). https://www.pentacam.com/fileadmin/user upload/pentacam.de/downloads/interpretationsleifaden/interpretation guideline_3rd_edition_0915.pdf

[19] M. Nejabat, “ORBSCAN Corneal Imaging” (Shiraz University of medical sciences). https://www.amedeolucente.it/public/Orbscan\%202.pdf

[20] Carl Zeiss Meditec AG, "Visante ${ }^{T M}$ OCT". https://www.epirusvisioncenter.gr/visanteoct.pdf

[21] J. Murua, "Cirugía actual de catarata," Rev. Med. Clin. Condes 21, 912-919 (2010).

[22] Department of Health and Ageing \& Medical Services Advisory Committee, "Optical biometry using partial coherence interferometry prior to cataract surgery," Barton Australia, Commonwealth of Australia (2005).

[23] B. Rra, S. Flaxman, T. Braithwaite, M. Cicinelli, A. Das, J. Jonas, "Magnitude, temporal trends, and projections of the global prevalence of blindness and distance and near vision impairment: a systematic review and meta-analysis," Lancet Glob Health 5, 88-97 (2017).

[24] J. Nichols, "2014 annual report-contact lenses 2014," Contact Lens Spectr. 30, 22-27 (2015).

[25] Ophthalmic Lens Market 2017 Global Analysis, Growth, Trends and Opportunities Research Report WiseGuyReports.com.

[26] J. Mountford, D. Ruston, T. Dave, Orthokeratology: Principles and Practice. United Kingdom, Butterworth-Heinemann (2004).

[27] M. Wang, Corneal Topography in the Wavefront Era: A Guide for Clinical Application. USA, SLACK Incorporated (2006).

[28] S. Barbero, "The concept of geodesic curvature applied to optical surfaces," Ophthalmic \& Physiological Optics 35, 388-393 (2015).

[29] F. Cavas, E. De la Cruz, J. Nieto, F. Fernández, D. Fernández, "Corneal topography in keratoconus: State of the art," Eye and Vision 3, 1-12 (2016).

[30] P. Harihar, Handbook of Optics 2nd Ed. USA, McGraw-Hill, Inc. (1995).

[31] D. Huang, E. Swanson, C. Lin, J. Shuman, W. Stinson, W. Chang, M. Hee , T Flotee , K Gregory , C. Puliafito, "Optical coherence tomography," Science 22, 1178-1181 (1991).

[32] M. Hee, J. Izatt , E. Swanson ,D. Huang , J. Schuman , C. Lin, C. Puliafito, J. Fujimoto , “Optical Coherence Tomography of the Human Retina," Arch Ophthalmol 113, 325-332 (1995).

[33] Y. Li, M. Tang, X. Zhang, C. Salaroli, J. Ramos, D. Huang, "Pachymetric mapping with Fourier-domain optical coherence tomography," J Cataract Refract Surg 36, 826-831 (2010).

[34] A. Fekry, A. Fayed, A. El Shayeb, A. Monem, M. Hamed, "Corneal epithelial thickness changes as imaged by Fourier-domain optical coherence tomography in eyes with keratoconus," Benha Medical Journal 22, 214-217 (2018).

[35] M. Brezinski, Optical Coherence Tomography: Principles and Applications. USA, Elsevier (2006).

[36] M. Kraus, Potsaid, M. Mayer, R. Bock, B. Baumann, J. Liu, J. Hornegger, J. Fujimoto, "Motion correction in optical coherence tomography volumes on a per A-scan basis using orthogonal scan patterns," Biomed Opt Express 3, 1182-1199 (2012).

[37] American National Standard for Safe Use of Lasers ANSI Z136.1-200.Laser Institute of America, American National Standards Institute, Inc. Orlando, (2000).

[38] J. Mobley, T. Vo-Dinh. Biomedical Photonics Handbook. Boca Ratón Florida, CRC Press LLC (2003).

[39] J. Kalkman, "Fourier-Domain Optical Coherence Tomography Signal Analysis and Numerical Modeling," Hindawi International Journal of Optics 2017, 1-16 (2017). 
[40] S. Yun, G. Tearney, J. de Boer, N. Iftimia, B. Bouma, "High-speed optical frequency- domain imaging," Opt Express 11, 2953-2963 (2003).

[41] M. Choma, M. Sarunic, Ch. Yang, J. Izatt, "Sensitivity advantage of swept source and Fourier domain optical coherence tomography," Opt Express 11, 2183-2189 (2003).

[42] I. Grulkowski, M. Gora, M. Szkulmowski, I. Gorczynska, D. Szlag, Susana Marcos, A. Kowalczyk,M. Wojtkowski, "Anterior segment imaging with Spectral OCT system using a high-speed CMOS camera," Opt Express 17, 4842-4858 (2009).

[43] S. Ortiz, D. Siedlecki, P. Pérez-Merino, N. Chia, A. De Castro, M. Szkulmowski, M. Wojtkowski, S. Marcos, "Corneal topography from spectral optical coherence tomography (sOCT)," Biomed Opt Express 3, 3232-3247 (2011).

[44] K. Bartlomiej, J. Kaluzny, M. Szkulmowski, M. Gora, M. Wojtkowski, “Corneal topography with highspeed swept source OCT in clinical examination,"Biomed Opt Express 2, 2709-2720 (2011).

[45] J. Wagner, D. Goldblum, P. Cattin, “Golden angle-based scanning for robust corneal topography with OCT," Biomed Opt Express 8, 475-483 (2017).

[46] S. Sayegh, "OCT corneal topography within $1 / 4$ diopter in the presence of saccadic eye movements," Proc. of SPIE 8571, 85713D-1- 85713D-7 (2013).

[47] S. LaValle. Virtual Reality. University of Illinois (2016). http://vr.cs.uiuc.edu/web.html

[48] R. McNabb, A. Kuo, J. Izatt, "Quantitative single and multi-surface clinical corneal topography utilizing optical coherence tomography," Optics Letters 38, 1212-1214 (2013).

[49] S. Das, Chi-Hao Liu, M. Shingh, M. Twa, K. Larin, "Modified wavelength scanning interferometry for simultaneous tomography and topography of the cornea with Fourier domain optical coherence tomography," Biomed Opt Express 9, 4443- 4458 (2018).

[50] R. Iyer, E. See, S. Mathews, "Smoothing splines on unit ball domains with application to corneal topography," IEEE Transactions on Medical Imaging 36, 518-526 (2017).

[51] D. Huang, Y. Li, M. Tang, Anterior Eye Imaging with Optical Coherence Tomography in Optical Coherence Tomography. Berlin Heidelberg New York, Springer [2008].

[52] Fedra Hajizadeh, Atlas of Ocular Optical Coherence Tomography. Cham, Switzerland (2018).

[53] P. Hariharan, Basics of Interferometry. Burlington MA, Elsevier (2007).

[54] D. Malacara, M. Servín, Z. Malacara, Interferogram Analysis for Optical Testing. Boca Raton FL, CRC Press (2005).

[55] J. Micali, J. Greivenkamp, "Dual interferometer for dynamic measurement of corneal topography," Journal of Biomedical Optics 21, 085007-1 - 085007-19 (2015).

[56] J. Micali, J. Greivenkamp, B. Primeau, "Dynamic measurement of the corneal tear film with a Twyman-Green interferometer," Journal of Biomedical Optics 20, 055007-1 -055007-11 (2015).

[57] J. Micali, J. Greivenkamp, "Method for reconstruction of complex surface shapes from a reflectionbased non-null interferometric measurement" Optical Engineering 55, 034101-1 -034101-14 (2016).

[58] T. Licznerski, J. Jaroński, D. Kosz, "Novel double path shearing interferometer in corneal topography measurements," Proceedings of SPIE 5959, 595917-1 - 595917-6 (2005).

[59] J. Espinosa, A. Roig, D. Mas, C. Hernández, C. Illueca, “Corneal topography reinterpretation through separate analysis of the projected rings," Proc. of SPIE 8427, 84273Q-1 - 84273Q-10 (2012).

[60] K. Sa, "A corneal topography algorithm that produces continues curvature," Optom Vis. Sci. 68, 957965 (1992).

[61] J. Snellenburg, B. Braaf, E. Hermans, R. Van der Heijde, V. Arni, D. Sicam, "Forward ray tracing for image projection prediction and surface reconstruction in the evaluation of corneal topography systems," Optics Express 18, 19324-19338 (2010).

[62] L. Carvalho, "Feasibility of a simple and cost-effective wide-angle corneal topography method for Placido-based Videokeratographs," Biomedical Optics and 3D Imaging OSA. (2012).

[63] A. Kilic, C. Roberts, Corneal Topography: From Theory to Practice. Amsterdam, Kugler Publications (2013).

[64] M. Rottenkolber, H. Podbielska, "Measuring ophthalmologic surfaces by means of moiré deflectometry," Opt. Eng. 35, 1124-1133 (1996). 
[65] H. Liang, E. Olesch, Z. Yang, G. Häusler, "Single-shot phase-measuring deflectometry for cornea Measurement," Adv. Opt. Techn. 5, 1-6 (2016).

[66] T. Dada. New Investigations in Ophthalmology, India, JP Medical Ltd. (2017).

[67] D. Kelly. Visual Science and Engineering: Models and Applications, New York, Marcel Dekker (1994).

[68] A. Kanellopoulos, G. Asimellis, "Color light-emitting diode reflection topography: validation of keratometric repeatability in a large sample of wide cylindrical-range corneas," Clinical Ophthalmology 9, 245-252 (2015).

[69] G. Fuentes, "Color Light Emitting Diode Reflection Topography- Clinical Applications," J Clin Exp Opthamol 9, 1-5 (2018).

[70] M. Campos, A. Estrada, R. Díaz, "New null-screen design for corneal topography," Proc. of SPIE 8011, 801124-1 - 801124-9 (2011).

[71] M. Campos, C. Cossio, V. Moreno, O. Huerta, "Surface shape evaluation with a corneal topographer based on a conical null-screen with a novel radial point distribution," Applied Optics 17, 5411-5419 (2015).

[72] M. Campos, C. Cossio, O. Huerta, V. Moreno, "Advances in corneal topography measurements with conical null screens, ” Proc. of SPIE 9628, 96281B-1-96281B-8, (2015).

[73] C. Cossio, M. Campos, "Accuracy and sensitivity analysis of the conical null-screen based corneal topographer," Proc. SPIE 9947, 99470Q-1 - 99470Q-8 (2016).

[74] A. Osorio, M. Campos, C. Cossio, "Characterization of a conical null-screen corneal topographer," Proc. SPIE 10330, 103301E-1 -103301E-11 (2017).

[75] V. Armengol, M. Campos, C. Cossio, "Evaluation of a human corneal surface with the null-screen Method," Proc. SPIE 10330, 103300Q-1 - 103300Q-8 (2017).

[76] M. Campos, V. Armengol, D. Aguirre, Ch. Camargo, "Obtaining the topography of human corneas with the null-screen testing method," Proc. SPIE 10692, 1069216-1 - 1069216-11 (2018).

[77] M. Rodríguez, A. Jaramillo, R, Diaz, "Dynamic point shifting with null screens using three LCDs as targets for corneal topography," Applied Optics 22, 6698- 6710 (2015).

[78] D. Malacara-Hernández, D. Malacara-Doblado, "What is a Hartmann test?" Applied Optics 54, 22962301 (2015).

[79] Y. Mejia, D. Malacara, "Object surface for applying a modified Hartmann test to measure corneal topography," Applied Optics 40, 5778-5786 (2001).

[80] Y. Mejia, J. Galeano, "Corneal topographer based on the Hartmann Test," Optometry and Vision Science 86, 370-381(2009).

[81] J. Schwiegerling, "History of the Shack Hartmann wavefront sensor and its impact in ophthalmic optics," Proc. SPIE 9186, 91860U-1 - 91860U-8 (2014).

[82] S. Goelz, J. Persoff, G. Bittner, J. Liang, Ch. Hsueh, J. Bille, "A new wavefront sensor for metrology of spherical surfaces," Proc. SPIE 1542, 502-510 (1991).

[83] J. Yang, X. Rao, Ch. Rao, "Measurement of Corneal Topography through Hartmann-Shack Wave-front Sensor," Proc. of SPIE Vol. 7160, 71601K-1 - 71601K-9 (2009).

[84] P. Mendoza-Villegas, G.Trujillo, D. Salas, A. Bustillos, "Method of analysis to measure the tangential curvature in a test sphere," Proc. of SPIE Vol. 7499, 74990K-1- 74990K-5 (2009).

[85] N. Yasumatsu, S. Watanabe, "T-ray topography by time-domain polarimetry," Optics Letters 37, 2706- 2708 (2012).

[86] S. Gruppetta, L. Koechlin, F. Lacombe, P. Puget, "Curvature sensor for the measurement of the static corneal topography and the dynamic tear film topography in the human eye," Optics Letters $\mathbf{3 0}$, 2757-2759 (2005).

[87] S. Brodie, "Corneal topography and the Hirschberg test," Applied Optics 31, 3627-3631 (1992).

[88] W. Frobin, E. Hierholzer, "Video Rasterstereography: A Method for On-Line Measurement of Body Surfaces," Photogrammetric Engineering \& Remote Sensing 57, 1341-134 (1991).

[89] G. Waring, Refractive Keratotomy for Myopia and Astigmatism. St.L.M. USA Mosby-Year Book (1992).

[90] H. Kaufman, Companion Handbook to the Cornea. Boston, Butterworth-Heinemann (2000).

[91] R. Arffa, J. Warnicki, P. Rehkopf, “Corneal Topography Using Rasterstereography," Eur J Implant Ref Surg 1, 45-48 (1989).

[92] M. Belin, "Intraoperative raster photogrammetrythe PAR Corneal Topography System" J Cataract Refract Surg 19, 188-192 (1993).

[93] S. Naufal, J. Hess, M. Friedlander, N. Granet, "Rasterstereography-based classification of normal corneas," J Cataract Refract Surg 23, 222-230 (1997). 
[94] D. Malacara, M. Servín, Z. Malacara, Interferogram Analysis for Optical Testing. Boca Raton FL USA, CRC Press Taylor \& Francis Group (2005).

[95] W. Chang, K. Chen, D. -Chin Chen, J. Tseng, S. Chen, Sun, J. Chen, Ken Y. Hsu, “Heterodyne moiré interferometry for measuring cornea surface," Optics and Lasers in Engineering 54, 232-235 (2014).

[96] Takeda M.; Mutoh K. "Fourier transform profilometry for the automatic measurement of 3-D object shapes,” Appl. Opt. 22, 3977-3982 (1983).

[97] J. Weber, 3D Reconstruction: Techniques, Analysis and New Developments. New York, Nova Science Publishers, Inc. (2016).

[98] D. Serrano, A. Martínez, J. Rayas-Álvarez, "Topography measurement of specular and diffuse surfaces," Proc. of SPIE 7792, 77921A-1- 77921A-8 (2010).

[99] D. Iskander, P. Wachel, P. Simpson, A. Consejo, D. Jesus, "Principles of operation, accuracy and precision of an Eye Surface Profiler," Ophthalmic \&Physiological Optics 36, 266-278 (2016).

[100] H. Nema, N. Nema. Diagnostic Procedures in Ophthalmology. India, New Delhi, JP Medical Ltd. (2014).

[101] L. Ventura, J. De Groote, S. Faria, H. Schiabel, “Corneal Topography for Slit Lamps,” IFMBE Proceedings 25/XI (2009).

[102] N. Garzón, F. Poyales, “ORBSCAN: Mapas topográficos,” Gaceta Óptica 420, 24-28(2007).

[103] C. Oliveira, C. Ribeiro, S. Franco, "Corneal imaging with slit-scanning and Scheimpflug imaging Techniques," Clin Exp Optom 94, 33-42 (2011).

[104] R.Martin, "Cornea and anterior eye assessment with placido-disc keratoscopy, slit scanning evaluation topography and scheimpflug imaging tomography," Indian J Ophthalmol 66,360-366 (2018).

[105] P.Lang, P. Thulasi, S.Khandelwal, F. Hafezi, J. Randleman, "Comparing change in anterior curvature after corneal cross-linking using scanning-slit and Scheimpflug technology," American Journal of Ophthalmology 191, 129-134 (2018).

[106] T. Li, L. Tian, L. Wang, Y. Hon, A. Lam, Y. Huang, Y. Wang, Y. Zheng, "Correction on the distortion of Scheimpflug imaging for dynamic central corneal thickness," Journal of Biomedical Optics 20, 056006-1-056006-9 (2015).

[107] C. Sun, H. Liu M. Jia, S. Chen, "Review of Calibration Methods for Scheimpflug Camera," Hindawi Journal of Sensors 2018,1-15 (2018).

[108] B. De Luis, J. Escudero, J. Pijoán, A. Santamaria, J. Etxebarria, "Evaluation of the Reliability and Repeatability of Scheimpflug System Measurement in Keratoconus," Cornea 37, 177-181 (2018).

[109] Y. Choi, Y. Eom, J.Suk, H. Myung, "Comparison of Anterior, Posterior, and Total Corneal Astigmatism Measured Using a Single Scheimpflug Camera in Healthy and Keratoconus Eyes," Korean Journal Ophthalmol 32,163-171 (2018).

[110] R. Ambrósio, "3-D Scheimpflug Corneal Tomography," Cataract \& Refractive Surgery Today, 2011, 42-44 (2011).

[111] F. Faria, R. Ambrósio, Clinical applications of the Scheimpflug principle in Ophthalmology, Rev Bras Oftalmol 75, 160-165 (2016).

[112] Zhe Xu, W. Li, J. Jiang, X.Zhuang, W. Chen, M. Peng, J. Wang, Fan Lu, M.Shen, Y.Wang, “Characteristic of entire corneal topography and tomography for the detection of sub-clinical keratoconus with Zernike polynomials using Pentacam," Scientific Reports 7: 16486, 1-10 (2017).

[113] A.Patel, R. Gouia, "Detecting topographical eye disorders," Biomath 5, 1-7(2017).

[114] B. Askarian, F. Tabei, A. Askarian, J.Chong, "An affordable and easy-to-use diagnostic method for Keratoconus detection using a smartphone," Proc. SPIE 10575, 1057512-1- 1057512-6 (2018).

[115] P. Keating, Geometric, Physical, and Visual Optics. Stoneham MA, USA, Butterworth-Heinemann (2002).

[116] V. Srinivasan, R.D. Thulasiraj, Ophthalmic Instruments and Equipment A handbook on care and maintenance. Madurai, India, Aravind Eye Hospital \& Postgraduate Institute of Ophthalmology (2003).

[117] J. Schwiegerling, Field guide to Visual and Ophthalmic Optics. Bellingham, Washington USA, SPIE Press (2004).

[118] H. Schswartz, Geometrical and Visual Optics A Clinical Introduction. New York, NY, USA, Mc Graw Hill Medical (2013). 


\section{Introduction}

The cornea is the first anatomical surface in the eye and contributes approximately $70 \%$ of the eye's total refractive power [1] due to: the change of refractive index between the air-tear film, anterior curvature, thickness and index change from cornea [2] to anterior chamber [3]. Some of its dimensions are shown in Table $1[1,4]$.

TABLE 1. Mean cornea dimensions.

\begin{tabular}{|c|c|}
\hline \hline Ocular parameters & Mean $(\mathrm{mm})$ \\
\hline \hline Vertical diameter & 11 \\
Horizontal diameter & 12 \\
Anterior surface curvature radius & 7.8 \\
Posterior surface curvature radius & 6.5 \\
Central thickness & 0.53 \\
Peripheral zone thickness & 0.71 \\
\hline \hline
\end{tabular}

*all dimensions are approximations and may vary from one individual to another.

Histologically the cornea from anterior to posterior surfaces is former by tear film $(4-7 \mu m)$, epithelium $(50 \mu \mathrm{m})$, Bowman's membrane $(8-14 \mu \mathrm{m})$, stroma $(500 \mu \mathrm{m})$, Dua's layer $(6.3-15.83 \mu \mathrm{m})$ [5], Descemet's membrane $(10-12 \mu \mathrm{m})$ and endothelium $(5 \mu \mathrm{m})$. The cornea can be arbitrarily divided into three zones of diameters: apical $(8 \mathrm{~mm})$, peripheral $(11 \mathrm{~mm})$, and limbal zone $(12 \mathrm{~mm})$ [6]. On the other hand cornea is an aspherical surface with positive spherical aberration and in a second order approximation can be modelled by a prolate ellipse [7] with an average normal asphericity value from -0.18 to -0.26 [8, 9]. The cornea is prone to develop some pathologies like keratoconus [10], ectasia [11] or Fuch's dystrophy [12] that affect its normal anatomical shape. In presence of these conditions, to determine the shape of the cornea is indispensable for an early stage diagnostic. For example, the measurement of the posterior corneal curvature (PCC) and the posterior surface topography is of utmost importance for the pre and post-surgical LASIK (Laser assisted in Situ Keratomileusis) evaluation. This is because the development of corneal ectasia [11] is a well-known complication in this type of surgeries [13]. It is known that ectasia could be attributed to unrecognized preoperative forme fruste Keratoconus $[14,15]$, if the shape and the location of the curve are outside the normal range and the central $5 \mathrm{~mm}$, eye may be suspect for keratoconus [16], for this reason evaluate PCC results imperative in every LASIK candidate. In addition, detection of corneal ectasia requires knowing the changes in sphericity of PCC and topographic anomalies, such as an increase in negative keratometric diopters [17]. Some of the commercial devices that can obtain elevation maps of the posterior surface are: Oculus Pentacam $\AA$ (Oculus) that is a slit-based system [18], Orbscan IIz® (Baush\&Lomb) based in Scheimpflug imaging [19], Visante® OCT-Atlas Corneal Topographer $®$ (Carl Zeiss Meditec) performs optical coherence tomography pachimetry and Placido disk elevation which together can provide posterior surface data $[14,20]$.

Nowadays, technological advances in medical science allow the surgical treatment of pathologies in the anterior segment of the eye. According to WHO (World Health Organization) [21], the cataract is responsible for $48 \%$ of cases of blindness worldwide, but can be successfully treated with surgical cataract removal and an intraocular lens implant; their implementation represents multimillionaire costs [22]. Refractive errors in the eye represents 53\% of all world's visual disabilities [23], affecting visual acuity and making necessary to add refractive components like ophthalmic or contact lenses to compensate these errors, both kind of lens is a market with a multimillionaire value [24,25]. An alternative treatment is the refractive surgery, which through a low power laser modifies permanently the shape of the cornea. In all of the above cases, obtaining reliable measurements in dimensions of the cornea is crucial and decisive for surgical planning or a correct lens prescription.

There are different kinds of methods to obtain corneal topography, some of them are currently implemented in available medical devices, and others have yet to reach commercial viability. In a general way, they can be classified into two groups according to the physical principle of operation: optical and nonoptical. Optical methods can be sub classified by the optic phenomenon used to measure the corneal surface: specular reflection, diffuse reflection, light scattering and image formation while non-optical are digital ultrasound and magnetic resonance imaging.

This paper presents a generic classification (Figure 1), trying to cover most of the optical techniques that have been reported in literature. Each method presents variants in modalities, configurations and set-ups, as well as advantages and disadvantages in such a way that it can be said that there is no perfect 
measurement method; they all have errors associated with limitations inherent to the technique and operation of the measurement system. This review does not mention non-optical methods like ultrasound digital topography or magnetic resonance imaging, besides it does not address the biometry by partial coherence interferometry and the measure of the posterior corneal is not covered in this paper completely.

\section{Surface maps of the cornea.}

The way in which some methods reconstruct the shape of the cornea is through a fitting process known as BFS (best-fit sphere); the fitting data comes from discrete points in the corneal surface obtained by measurements of the different techniques. For example, elevation data is obtained after subtracting the fitting to a reference surface. Generally, the result of this type of fitting is the deployment of several types of maps briefly described in this section in a qualitatively manner.

- Map of axial curvature. In this type of map, the anterior curvature is represented. These maps are obtained by measuring the curvature of the cornea at each point relative to a specified axis that is typically the visual axis. This map is dependent on the assumption that the center of curvature at the surface is located along the optical axis in such a way that the radius is called the radius of axial curvature.

- Map of tangential curvature or instant map. The radius of tangential curvature indicates the local curvature of the cornea in the meridional plane; the reference axis is different for each point.

-Gaussian curvature. This curvature is defined as the geometric mean of the maximum and minimum curvature of a specific point on the corneal surface. Gaussian curvature maps are not dependent on the fixation or symmetry of the cornea and show the local curvature independently of the axis; they are very useful in the exact determination of the location and effect of surface irregularities.

-Maps of elevation. This map displays the sagittal height $(\mu m)$, in relation to a reference plane. Regularly this plane is a sphere that resembles the general diameter of the cornea under study. Each point on the surface that matches the reference sphere is represented by green colors; warmer colors represent points higher than the reference plane, while colder colors represent points that are lower than the reference plane. Changes in elevation represent refractive power. These maps are very useful to evaluate the shape of the cornea with respect to a smoothed spherical surface. The importance of these maps for example in refractive surgery is the ability to predict the effect that tissue removal will have on corneal elevation [26, 27].

- Pachymetry maps. It provides the measurement of the distance from the anterior to the posterior surface in a direction perpendicular to the anterior surface, also giving information on the distribution of thickness along the surface.

-Refractive map. This map displays the refractive power of the cornea, which is calculated based on Snell's law of refraction, assuming the optical infinity. This map correlates the shape of the cornea and vision, thus being very useful for understanding the effects of refractive surgery.

- Wavefront maps. These maps describe the optical path difference between the measured wavefront (in the input pupil) of the corneal surface and a reference wavefront. The wavefront error is obtained mathematically from the reconstructed wavefront trough different techniques such as: peak-peak error, root least squares error (RMS), Strehl relation, point spread function (PSF), modulation transfer function, phase transfer function, and optical transfer function. Like the elevation maps, each color represents a specific degree of error in the wave front measured in microns and it is necessary to consider the range and intervals of the scale.

-Maps of differences. The difference map displays changes in certain values between two maps. It is used to monitor any type of change induced by contact lenses or by refractive surgery.

-Regular map. This map displays some values comparing them with an arbitrary standard and with a specific mathematical model. Magnify unique features of the cornea under inspection.

-Maps of surface quality. This map uses the same technique as the elevation map but takes as its reference surface the cylindrical spherical surface of better fit. The difference between the theoretical surface and the subject to inspection represents the part of the cornea that cannot be corrected optically and just as the refractive map only has clinical significance when the values considered are within the pupillary area. 
ÓPTICA PURA Y APLICADA

www.sedoptica.es

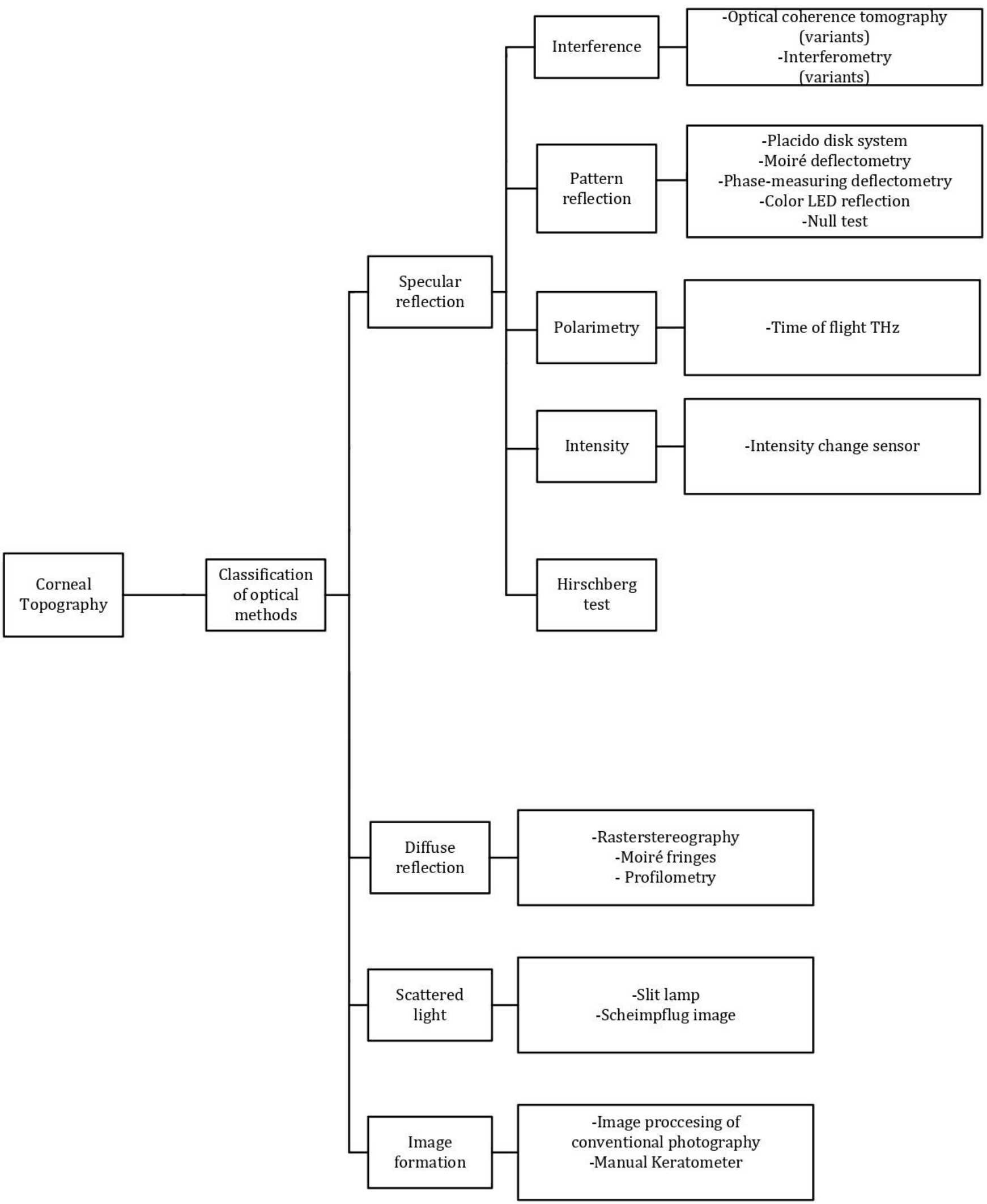

Fig.1. Classification for corneal topography measuring methods. 
Some of the algorithms used to obtain this type of maps assume that the normal at each point on the corneal surface lies in the meridional plane, so that not necessarily a point of interest will lie in this plane. A recent study proposes the use of geodesic curvature maps [28], which is a measure of how far a part of a specific curve is from the beginning of a curvature line and its corresponding geodesic line. These maps can be used for the local axial characterization of asymmetries in corneal topography offering a better sensitivity than the radial curvature to locate axial asymmetries. Other results of the different topographic systems are the indices that allow quantifying the level the level of irregularity of the surface. These indices cannot be extrapolated between different topographic systems because the degree of specificity is unique to each system from which it comes. Cavas et al [29] presents a detailed description of these indices.

\section{Specular reflection techniques}

\section{3. a. Interference}

The interference phenomenon has been used to perform a wide range of precision measurements by means of optical interferometers [30]; these are devices that are used to produce interference patterns between two or more waves. Usually the waves are extracted in some way from a single source of light. Interferometers can be classified according to the method used to produce the waves from the light source. There are two types: by amplitude and wavefront division. The measurement of the cornea by interference phenomenon has been studied using different techniques, arrangements and configurations that will be described in the following two sub sections.

Optical coherence tomography (OCT) [31] is used in clinical ophthalmology in applications such as retina images, pachymetry, and elevation maps of the epithelium [32-34], but remains limited in corneal topography measurements. The OCT can be divided into two types, one based on the time domain (TD-OCT) and the Fourier domain (FD-OCT). In the case of TD-OCT, the most commonly used optical configuration is a Michelson interferometer (Figure 2). This type of interferometer consists of a beam of light, which falls on a beam splitter (BS) that leaves pass part of the light and reflect the other, in such a way that it is divided into two arms, one of reference and the other of test. The reference beam is transmitted and propagates until it meets the mirror M1 where it is reflected back to the beam splitter, in the beam splitter this wave is split in two waves, one transmitted which return to the light source and another reflected one that leaves in the direction of the exit port (detector). Something similar happens with the test beam, it is reflected in the beam splitter, it will travel towards the surface under test where it will be reflected towards the beam splitter and part will be transmitted to the detector capturing the interference signal. The waves reflected through the different surfaces of the sample under test are delayed in time with respect to those reflected by the reference mirror.

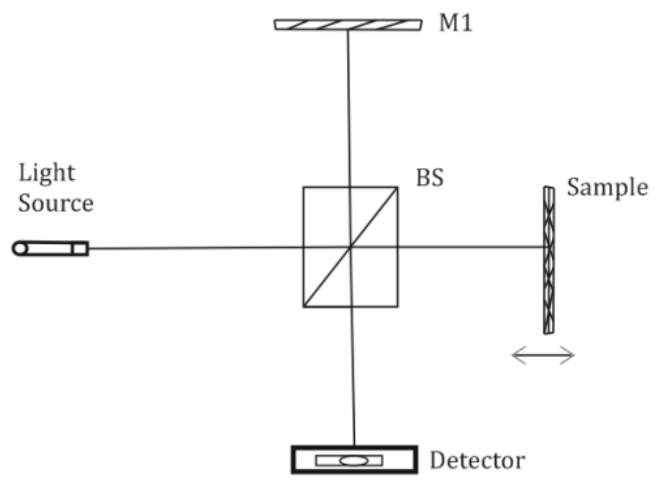

Fig.2. Time-domain OCT system.

Doing an analysis of an aligned interferometer, the intensity in the detector is given by equation 1 [35]:

$$
I_{\text {detector }}=I_{r}+I_{s}+2 \operatorname{Re\gamma }(z)_{11} \sqrt{I}_{r} I_{s} \cos (2 k \Delta L) .
$$

where $I_{r}, I_{s}$ are reference and sample arms intensities respectively, $\gamma(z)_{11}$ is a normalized function, cosine argument $2 k \Delta$, is pathlength difference. It can be seen that when $k \Delta L=2 N \pi$ a maximum is obtained and if $k \Delta L=(2 N+1) \pi$ a minimum will appear. In order to obtain an axial profile of the sample, the mirror in the 
reference arm M1 will move with a velocity $v_{m}$, so there is going to be a frequency shift in the signal. Applying frequency shift to eq. 3 cosine argument will change to 2 :

$$
I_{\text {detector }}=I_{r}+I_{S}+2 \operatorname{Re\gamma }(z)_{11} \sqrt{I}_{r} I_{S} \cos \left(2 \pi f_{D} t\right)
$$

A cosine signal is obtained when the difference between the optical path lengths of the reference mirror coincides with some layer of the surface under test. The envelope of the interference pattern provides the axial profile of the sample. To obtain the three-dimensional profile, a sweep of the reference light beam or of the surface under test is performed (which is a disadvantage of the TD-OCT, due to the low feasibility to obtain real-time images). At the same time, a scan can be performed with the reference mirror to profile the test surface axially. The different scans can be classified into types A, B and C [36]. The equation 4 is a general description of the interference in a Michelson interferometer assuming ideal conditions and just for one dimension, but solving for two dimensions and with a specific spectral source profile is developed in order to obtain low coherence interference that implies an a more complex development [35]. Generally, are used sources with wavelengths between 800-850 and $1310 \mathrm{~nm}$ with bandwidths of up to $5 \mathrm{~nm}$, according to the exposure limits established by ANSI [37]. The reason for use these wavelengths is the minimal absorption in organic tissues, due to the amount of water they contain according to the therapeutic window [38].

The FD-OCT [39] uses the same operating principle of low coherence interferometry and is generally implemented with a Michelson interferometer shown in Figure 3, this OCT systems are based on a measurement of the interference spectrum ether in space on a spectrometer (spectral domain) or in time with a wavelength sweep (swept-source). The basic operation is similar to that described above. For example, in the case of spectral domain, assuming a test sample with two surfaces $\mathrm{c} 1$ and $\mathrm{c} 2$ on reflection the incident beam will cause an OPD L1 for the first surface and another OPD L2 for the second surface. The reflected light from the two surfaces is combined in a dispersion grid that angularly separates the different wavelengths and by means of the $\mathrm{d} 2$ lens, it will focus on a linear CCD the interference pattern that is formed from the superposition of the reference surface and the two surfaces of the sample. Because the phase difference depends on $\bar{k}$, a modulation will be introduced into the intensity spectrum. If the sample consists of $N_{i}$ layers, the signal of each layer will interfere with the reference signal because the difference of optical paths of the interferometer will coincide with the difference of optical paths between the points reflected by the sample, producing a modulation in the intensity spectrum, whose frequencies will be proportional to the OPD between the reference mirror M1 and the $N_{i}$ layers of the sample. The Fourier transform of the intensity pattern captured in the CCD results directly at the modulation frequencies and thus at the position of the $N_{i}$ reflecting layers with respect to the reference surface. With the FD-OCT it is only necessary to take one shot, being able to obtain images through a type B scan, in such a way that the mobile parts that have a TD-OCT are no longer necessary, which represents a great advantage. Is important to note that in most of the experimental and commercial arrangements in applications of FD-OCT for eye images are based on interferometers with fiber optic couplers, so it is very common to find in the literature set-ups that are not built with bulk optics components.

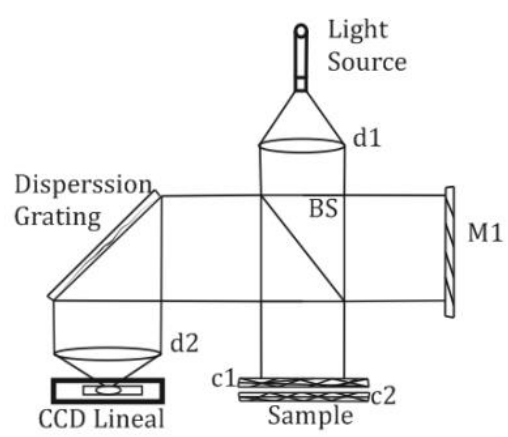

Fig.3. Fourier-domain sOCT system.

The application of the OCT for obtaining the corneal topography is limited because it has several disadvantages. One of the disadvantages of OCT systems is the speed of acquisition of the image. Large mapping times can result in incomplete scans or artifacts and disturbances due to abrupt movements of the eye and involuntary blinks. Thus, different modalities of the OCT have been implemented, such as the swept 
source OCT (ss-OCT) or the spectral OCT (s-OCT). The ss-OCT, works by encoding the wave number in time by adjusting a narrow band source along a given bandwidth [40] obtaining a better sensitivity than the TDOCT [41] and with a scanning speed able to obtain images of the anterior segment of the eye in real time. [42]. Another problem that affects the OCT is field distortion that causes flat surfaces in the image to appear as curves, this is attributed mainly to the configuration of the scanning set-up, being affected by the spatial separation of the mirrors of the scanner and the design in alignment of the lenses with respect to the scanner's mirrors. Compensation solutions have been applied to this problem, yielding good results with estimations of asphericity close to the values of the gold standard [43]. The ss-OCT technique applied to corneal topography has certain advantages when evaluating corneas with complex topographies (cornea after penetrating keratoplasty) because it provides tomograms with a more homogeneous retro-scattered intensity distribution where the application of segmentation algorithms does not assume the smoothing of the corneal surface as in other methods [44].

Most topographic maps obtained by ss-OCT are generated from a parametric representation of the reconstructed surface by the use of Zernike polynomials. Because these polynomials lose orthogonality by scanning at discrete locations distributed over the cornea area, the use of certain scanning patterns directly influences the numerical conditions of the reconstruction with polynomials. Recently Wagner-Catin et al [45] proposed a scan pattern based on the golden angle where they reported the possibility of decreasing repeated measurements, due to its proposal of distributed area coverage, abrupt disturbances can be easily detected and excluded from reconstruction in order to obtain a reliable topography even in measurements acquired under non-optimal conditions.

During the last 10 years other methods have been developed for the determination of corneal topography based on OCT as proposed by S. Sayegh [46] predicts corneal curvature by starting from the type B scanning rate and the velocity of saccade eye movement [47] peak in (m/s). However, in order to be feasible, scanning speeds of $300 \mathrm{kHz}$ are needed, besides the geometric model used is not useful in pathological anatomies of the cornea because when the curvature varies abruptly is necessary to increase the scanning speed and is not possible because of the commercial OCT scanning speeds.

Mc Nabb [48] proposes a more accurate approximation to the standard for corneal topography. He adopts as reference the topographic corneal axis to obtain the axial map and the meridional curvature as a function of the elevation of the corneal profile with respect to corneal topographical distance axis, calculating analytically by model surface profile as a fifth order Zernike polynomial. The obtained results were comparable with those retrieved with two other corneal topographer systems based on Placido rings and photography of Sheimplflug. In the pilot study all volumes were acquired with a distributed scan that performs linear A-type scans over time but not in space, thus generating sub-sampled and temporally separated profiles of the sampled radial meridian. With this method, it is suggested that by means of optical coherence tomography with a distributed scan it is possible to perform ketarometric measurements using commercially available s-OCT systems.

S. Das et al [49] reported the implementation of an algorithm of interferometry by scanning at wavelength (mWSI) that was applied with a traditional FD-OCT scanning system. They used ex vivo pig corneas and reconstructed the topography at an artificial intraocular pressure of $10 \mathrm{mmHg}$. The developed technique focused more on demonstrating the precision of the technique in measurements of the roughness of the corneal surface and not so much in the topography.

R. Iyer et al [50] presents a method to reconstruct the corneal topography from elevation data of the anterior surface obtained from OCT images of a keratoconic patient along degree planes of $0^{\circ}-180^{\circ}, 45^{\circ}-$ $225,90^{\circ}-270^{\circ}$ and $135^{\circ}-215^{\circ}$ that they collected manually with 64 points. The resulting surface was the sum of two functions that exist in two sub spaces $\left(\mathcal{H}_{0}, \mathcal{H}_{1}\right)$ orthogonal within a set of differentiable and integrable equations; the first sub-space contains a set of Zernike polynomials as base function while the second contained asymmetric radial functions. With a solution focused on a penalized weighted least square regression (PWLSR) with a penalty on magnitude equal to the Laplacian of the surface. The authors presented as results the reconstruction of the corneal surface with a residual error (RMS) at the measured points of $23.5 \mu \mathrm{m}$ and where the ectasia is clearly seen. They also presented the representation of the surface with a band of $95 \%$ confidence with widths confidential average of $78 \mu \mathrm{m}$ and the prediction band of $164 \mu \mathrm{m}$.

Even though OCT is a technique widely used to obtain images of the anterior segment of the eye [51], it is not a gold standard for measuring corneal topography; proof of this is that to our knowledge the commercial 
equipment to date based on this technique that includes a complete measurement of corneal topography [52].

One of the most commonly used interferometers for the measurement of corneal topography is the Twyman Green interferometer (TGI) [53] its basic configuration is shown in Figure 4. A TGI basically is a Michelson interferometer illuminated with collimated light in such a way that fringes of equal thickness are obtained. With the configuration of this interferometer the optical paths can be almost equal so that interference fringes can be obtained with a good visibility having a source with a limited coherence length. The light coming from the laser is expanded and collimated by a microscope objective and a collimator lens. To obtain a flat wave front without diffraction rings in the field, a spatial filter (pinhole) is placed. One of the main applications of this type of interferometer is in optical tests for components such as parallel flat plates, prisms and lenses [54]. The use of this interferometer to obtain the corneal topography is because the cornea acts as a convex mirror, the pattern of fringes can be interpreted as a contour map of surface elevation when the difference between two consecutive strips is equal to the elevation difference of half the wavelength used. A topographer based on interferometry can be called an interferometric corneal topographer (ICT).

Recently D. Micali et al. [55] reported the reconstruction of the corneal topography using a pair of polarization-splitting Twyman- Green interferometers by polarization division. This interferometer had a dynamic range of surface of $256 \mu \mathrm{m}$. They used the space carry method to measure the phase in a single image. In spite of having a reduced signal modulation by several effects such as tilt and defocusing caused by eye movement, an [56] unwrapping algorithm guided by the quality of the phase was successfully implemented. In addition, the reconstruction was subjected to a process for correcting the delay error [57] introduced by the non-null configuration used in the interferometer. This type of error is the result of the disturbance of the test wavefront that propagates through different sections of the interferometer. They developed a method to calculate the absolute position of the test surface relative to the interferometer or to a known point in space based on the measured amount of defocusing.

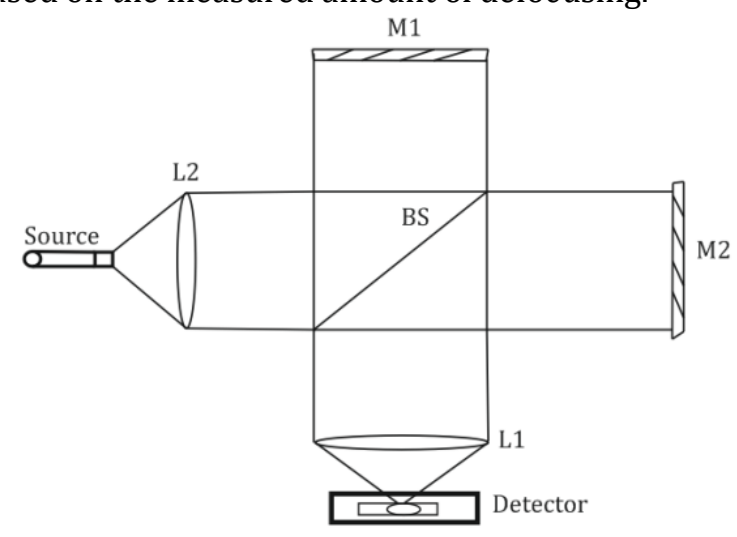

Fig.4. Twyman-Green interferometer system.

Ten test surfaces were used to validate the radius of curvature obtained, improving the radii obtained with the Placido ring technique by an order of magnitude. At the time of obtaining the corneal topography they demonstrated that the ICT can solve the high and medium frequencies that correspond primarily to the tear film, thus adding another advantage since if the residual surface (after removing a biconical surface) results in a surface flatter would have resulted mostly in noise. The built ICT has a spatial resolution of $6 \mu \mathrm{m}$ an absolute height resolution of $5 \mu \mathrm{m}$ and a resolution of $25 \mathrm{~nm}$ in relative height, which exceeds the resolution and precision of commercial topographic systems based on Placido rings.

T. Licznerski et al. [58] reported a double path shearing interferometer (DPSI), that is, they captured the interference patterns simultaneously in two orthogonal directions. The DPSI consisted of two independent arrangements that work as lateral shear interferometers. In each interferometer the beams are laterally displaced and there is an inclination introduced between them in such a way that the interference pattern captured by the CCD corresponds to the difference of optical paths between the wavefronts created by the lateral shear and the relative slope between interfering wave fronts. With two ultrasonic sensors they knew the position of the eye and maintained an appropriate distance between the eye and the objective in the experimental arrangement. The slope between the interfering wave fronts results in the carrier frequency 
of the fringes in the interferogram, in such a way that measurements of the phase difference in $x$ and $y$ axis can be obtained. Applying the fast Fourier transform they obtained the differences between the wave fronts of the captured interferograms, after discrete integration of the phase differences along the lateral shear and slope introduced in two perpendicular directions, they obtained the wavefront reconstruction. Although they demonstrated the reconstruction of the corneal topography with a more complicated interferometry configuration than the TGI, they did not report comparisons of their measurements with respect to other measurement systems.

\section{3.b. Pattern reflection}

Within this classification are several methods that are described below.

About Placido system owes its name to a Portuguese oculist named Antonio Placido who invented the keratoscope in 1880. The Placido system projects the image of a set of illuminated rings on the cornea Figure $5 \mathrm{a}$, the image of the rings is formed approximately $4 \mathrm{~mm}$ after the corneal apex as shown in Figure $5 \mathrm{~b}$. The image of the illuminated rings is captured by digital camera and sent to a computer for processing. The way to determine the radius of curvature of the cornea is through the transitions of the reflected bright and dark rings, the shape and its size give information on the local curvature of the cornea. In the reflected image the smaller the distance of a ring to the center of all the rings the greater curvature of the cornea and inversely the larger the distance, smaller the curvature.

Like all systems for measuring corneal topography, Placido systems also have limitations. One of them is that this technique is based on the calculation of the radius of curvature by the changes observed in the rings of the reflected image. A correct detection and classification of these rings is essential for an adequate representation of the corneal surface. But one of the biggest problems of this system is that in some cases the algorithms used for the reconstruction carry out a conversion of cartesian coordinates to polar coordinates assuming the symmetry of the rings. However, the rings are not completely symmetrical because the corneal surface is not, in addition to not having the same center coordinates, the cornea and the rings are not on the same axis. This lack of circular symmetry leads to distortion and loss of information because the data is interpolated in conversion coordinates. As a solution to this problem, different alternatives have been proposed to minimize these effects.

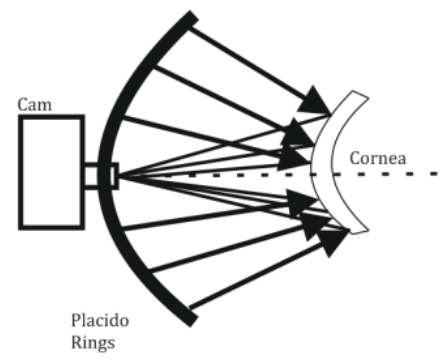

a)

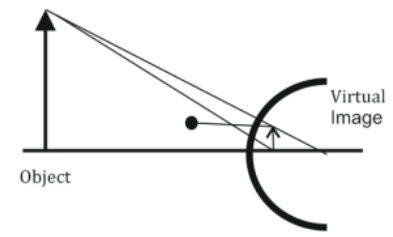

b)

Fig.5. a) Placido disks projection on the cornea, b) Virtual image formed by Placido disks.

J. Espinosa et al [59] presented an elliptical scanning algorithm that minimizes the error of the sum of the squares of each of the scanned rings and managed to avoid the angular sampling interpolation that is caused by the conversion of cartesian to polar coordinates. The first thing that the algorithm performs is a high pass filtering with top-hat operations of erosion and expansion to have a better contrast in the image. Then using a Canny methodology, they found the edges of the rings and with the elliptical scanning algorithm they obtained two edges for each ring. As results, they first verified the consistency of the method by measuring the radii of curvature of the calibration spheres used in the study, finding a correlation coefficient of $r^{2}>0.99$, which proved a good reproducibility of the algorithm. It demonstrated to allow the analysis in annular regions of the cornea managing to reduce the density inequality of samples in different sectors of the tested surface allowing the improvement of the fitting to reference surfaces.

Another of the most significant disadvantages of the Placido ring system is the skew ray error. It is caused by the fact that the light that comes out from Placido rings tends to diverge. Thus, is not possible to know the corresponding point between the source and an image point, because it is assumed that the point of the 
source is contained in a meridional plane, which is only true for symmetrical rotational surfaces. Several algorithms to obtain curvature are available and used along with this technique, an example is the Arc Step [60] implemented by backward ray tracing, but due to the cornea's asymmetrical surface, skew rays can introduce significant errors in the reconstruction.

Snellenburg et al [61] proposed a solution to the previous problem. They used an algorithm of ray tracing that performed better than the basic arc step algorithms that often are imprecise to determine correctly quadrafoil aberrations.

Another problem with this technique is the lack of coverage of the entire area of the corneal surface, present in devices based on this method. Giving a complete map of the entire cornea is important when prescribing contact lenses. To solve this problem Carvalho [62] proposed an adaptation of image acquisition at a certain angle using a commercial Placido topographer. The information of each image was stored in cylindrical coordinates and after the elevation calculus of the 9 different projection positions, the cylindrical matrices were transformed to cartesian coordinates. In order to be able to implement the translations and geometric rotations placing the same axis of reference in all the matrices, in such a way that they obtained 9 maps oriented to the same axis of reference, resulting in a conventional map with values at all measuring points of the cornea. Using conventional algorithms performed a fitting using Zernike polynomials joining all points of the 9 maps in one map and thus able to represent twice the area than a conventional topographic map, however, they not presented a comparison between cornea elevation maps because they did not have other methods to compare their results.

Other disadvantages are that the image of Placido rings is formed by the reflection of the tear film, so, any irregularities in the thin layer affect the accuracy and quality of the acquired image. This we must add that should have an intact epithelium. Furthermore, due to the nature of the Placido rings, the exact center of the cornea cannot be measured directly, but through an extrapolation based on the surrounding areas the center is approximated [63]. Another limitation is that it does not obtain information about the posterior surface of the cornea, so is not possible to obtain pachymetry maps. There are variants of the Placido ring pattern that use the projection of a polar mesh that offer measurements of the surface up to the limbal zone and provide data of radial and concentric points, allowing measurements of radial distance and concentric rotational changes in the cornea.

On the other hand, Moiré deflectometry consists in the formation of a pattern when two grids are placed at a small angle between them, separated by a distance $d$ as shown in Figure 6a. Moiré deflectometry, uses two identical Ronchi grids with a certain separation $p$ between their lines. When these are exactly parallel you can see the infinite bright or dark lines. If a small angle is induced between the grids will produce a pattern of separate stripes. The separation $p$ " between the segments can be denoted by the following equation 3 :

$$
p^{\prime \prime}=\frac{p}{2 \sin (\theta / 2)}=\frac{p}{\theta}
$$

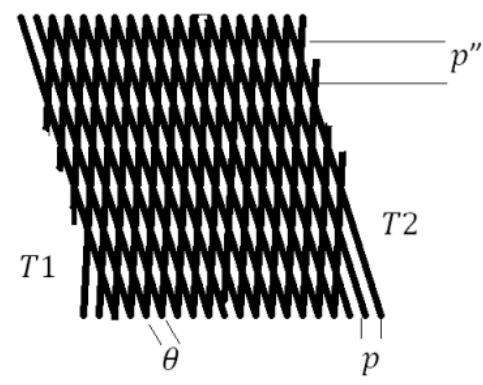

a)

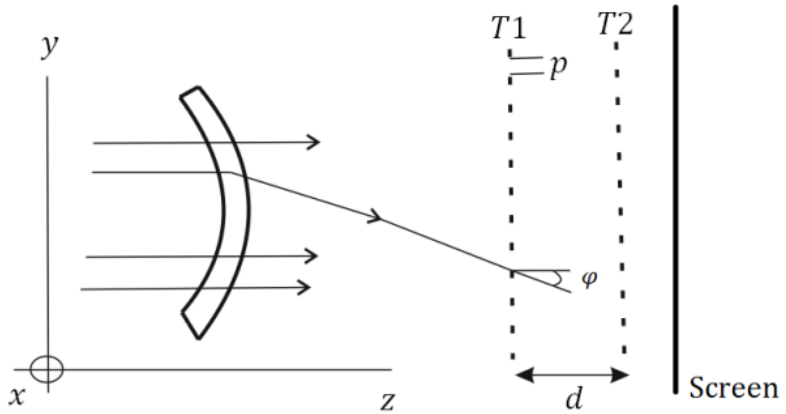

b)

Fig.6. a) Moiré effect produced by two identical grids, b) distorted beam modifies Moiré fringes pattern.

The way in which this phenomenon is used to make measurements is placing a test object (Figure 6b) which will be a phase object located between a collimated beam source and the grids. When the beam of collimated light passes through the object, in this case the cornea, the wavefront will be modified causing the image of the first and second grid to be distorted thus changing the Moiré pattern. From the displacement $h$ of a strip 
with respect to a reference position, it is possible to know the deflection angle, and can be denoted by the following expression 4 :

$$
\varphi=\frac{h \theta}{d}
$$

where $d$ is the distance between the two grids. This is known as the finite mode Moiré deflectometry. There is also the infinite mode in which $\theta=0$, and therefore the corresponding angle of a strip is:

$$
\varphi=\frac{p}{d}
$$

A deflection map of the rays in the direction normal to the gratings will be observed with increments of $p / 2$. Since $\varphi \propto 1 / \mathrm{d}$ increases, the distance between the grids, sensitivity increases, and a periodic phenomenon appears where the Moiré pattern is focused, blurred and then refocused. The distance in which the pattern is focused is known as the Talbot image planes and occurs when $d=m p^{2} / \lambda$ for $m=0,1,2,3 \ldots$.The increase in angular resolution causes a reduction in spatial resolution by diffraction effects. The spatial resolution $\delta_{\varphi}$ in each plane is $m p$ and the angular resolution $\delta_{\varphi}$ is $(\lambda / m p) q$ where $q$ the number of fringes that can be resolved. The multiplication of these two quantities yields the uncertainty principle in optics $\delta_{\varphi} \delta_{x}=\lambda q$, which means that this method is limited by diffraction.

The only work reported in the literature applying the Moiré deflectometry to the measurement of corneal topography is the one reported by Rottenkolber et al [64]. They report obtaining a curvature radius of a human cornea with results comparable to those obtained by a commercial keratometer, in addition to finding a distortion distribution similar to the results obtained by a TGI.

There are a variant of Moiré deflectometry, Liang et al [65] proposed the use of deflectometry-phase measurement for the reconstruction of the corneal topography. This technique works by pixel-by-pixel analysis of the phase in the fringes of an image reflected by the surface under test, usually a sinusoidal pattern is projected. With this information it is possible to calculate the local gradient of the surface by deducting the curvature of the derivation and the topography of the integration. They used an experimental arrangement with geometry aligned within the axis, using a plane mirror of $0.4 \mathrm{~mm}$ mounted in the center of a $26^{\prime \prime}$ LCD monitor, located at $100 \mathrm{~mm}$ from the cornea and with slope range of $29^{\circ}$. The image of the pattern reflected by the mirror incised to the camera that was next to the screen. The pattern was determined experimentally, being the maximum possible frequency of a period in the reflected image of 12.5 periods per millimeter. Preliminary results that they obtained with a test volunteer diagnosed with $1.25 \mathrm{D}$ of astigmatism, was a deviation from $0.15 \mathrm{D}$, which suggests an encouraging result, which can be improved by correct longitudinal positioning of the cornea by a method that uses an auxiliary camera to measure the position. The main advantage of this method is that by capturing a single shot you can carry out all the processing and measurements.

In relation to Colour LED reflection [66] this method works with the principle of point-to-point ray tracing combined with the second Purkinje image [67]. Its projects over a corneal area of $8.5 \mathrm{~mm}$ around 700 LED red, green and yellow sources. This technique has sub micrometric accuracy greater than $<0.8 \mu \mathrm{m}$. The principle of operation is triangulation. The main advantage is the time of capture of the image since is not affected by artifacts of eye movements. J. Kanellopoulos [68] in his study concluded that this technique offers a high specificity for the determination of cornea irregularities. G. Fuentes [69] recently reported that there is a strong correlation between the values of astigmatism and SimK of an equipment based on this technology and another based on Slit-Scan technique.

What concerns to null tests basically consist of a direct representation of the deviations of the measured surface with respect to an ideal surface and the calculation of the variable of interest is carried out based on the differences between both surfaces.

M. Campos et al. [70-75] proposed a new null test design based on a conical screen. With an arrangement of points in shape of a drop, by reflecting these on the surface under test appear in the form of an ordered arrangement of points in an image that is captured with a CCD camera, with an experimental arrangement shown in Figure 7. The rays coming from the reflection pass through a small opening (pinhole) of such so that the image is formed in a dark camera environment. The opening was large enough to avoid diffraction effects even though the developed test is in the regime of geometrical optics. With a positive lens, the rays are collected to focus on the CCD sensor. The effects of spherical aberration and coma induced by the positive lenses of the camera are considered in the model while the distortion is minimized with a previous calibration of the system. With the intention of improving the accuracy of the technique, they modified the 
design of the null screen, increasing the number of sampled data. For the design of the null screen, they considered a cone with radius $s$ and height $h$ oriented along the $z$ axis, the distance $d$ on the CCD sensor and the diameter $D$ of the surface under inspection.

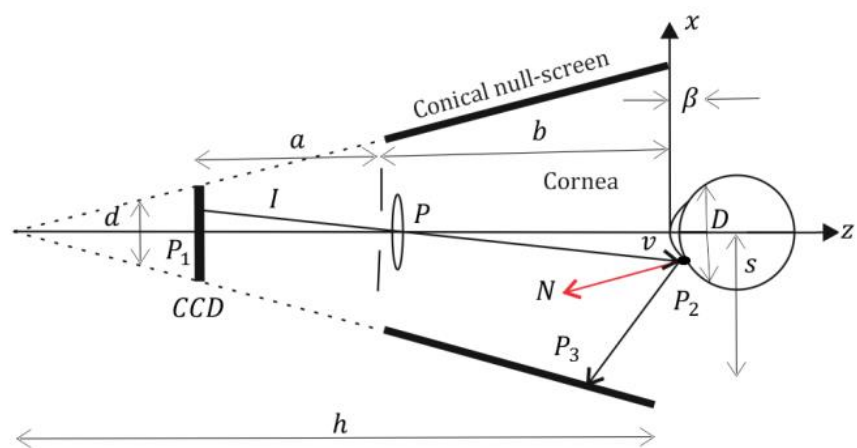

Fig.7.Set up for the null test proposed by Campos et. all.

By means of a transformation of cylindrical to Cartesian coordinates, $(x, y)$ they printed the null screen with a laser printer. The method to obtain the shape of the surface integrates the Cartesian components of $N$ normal vector to the surface under test and making a Levenberg-Marquart fitting of the experimental data to a proposed aspheric surface. In the last publication [76], it reported the change of the screen to its negative modality because it allowed them to have a better coverage of the corneal surface. They evaluated the conical corneal topographer in human corneas of two different individuals and comparing it with the results obtained by a commercial corneal topographer. Elevation maps, tangential maps, sagittal maps and curvature maps were generated where very similar results were obtained to the commercial corneal topographer for the subject 1 . In this study, they concluded that their topographer could obtain the reconstruction of the shape of the corneal surface with an accuracy of $5.9 \mu \mathrm{m}$ to $20.3 \mu \mathrm{m}$. However, they recognized the need to redesign the test casing for use with different morphologies of the human face and thus avoid misalignments that affect the measurement, as was the case with test individual 2, where the results obtained greater differences than commercial topographers. An advantage of this proposal is that it not requires specialized optical design, which makes it cheap and easy to implement.

Rodriguez et al. [77] investigated the use of LCD technology as null screens in a triangular prism configuration. Using the reverse ray tracing method, they calculated the position of the points on the screens in such a way that the image reflected by the test surface resulted in a perfectly ordered grid arrangement. The dynamic point shifting (DyPoS) method is applied to these points, which consists in changing the position on the null screen and taking an image in each position. The results of this technique showed a $3 \%$ error in the measurement of the radius of curvature of a calibration sphere. Although this method has not been quantitatively tested on a human cornea, it has some advantages such as the possibility of the deployment of different patterns and geometries, as well as the variation of the number of points in the projection, which would increase the resolution and accuracy of the technique. Another advantage is that the use of LCDs is safe for the human eye. A disadvantage presented by this method is the inhomogeneity in the illumination of the patterns since experimentally areas with low intensity were identified, which results in the difficulty to distinguish and separate the points in order to obtain their centroids.

Another technique used in ophthalmology to measure corneal topography is the quasi-quantitative Hartman test [78]. This test can be implemented as a null test. It uses geometric optics as a basis for the approximations of the reconstruction of a wavefront that is sampled discreetly on a reflecting surface. The wavefront is obtained by Newton's integration of transversal aberrations joining the sampled points. Arrangements of the holes for the test can be square, radial or helical. By Rayces equations is possible to find the relation between the aberrated wave front $(W)$ and the deviation of the ray with respect to the ideal ray position with the following equation (6):

$$
\frac{\partial W}{\partial x}=\frac{\Delta x}{d}
$$


where $\Delta x, \theta_{x}$ is the angular deviation and $d$, the distance between the surface and the projection plane (Figure 8). In addition, the longitudinal spherical aberration (LSA) associated with the ray passing through the edge of the pupil at height $R$ is given by (7):

$$
L S A=n\left(\frac{1}{m}-\frac{1}{d}\right)
$$

where $m$ is the longitudinal distance from the pupil to the intersection of the marginal ray, $R$ height of the half pupil, $n$ refractive index in the object or image space.

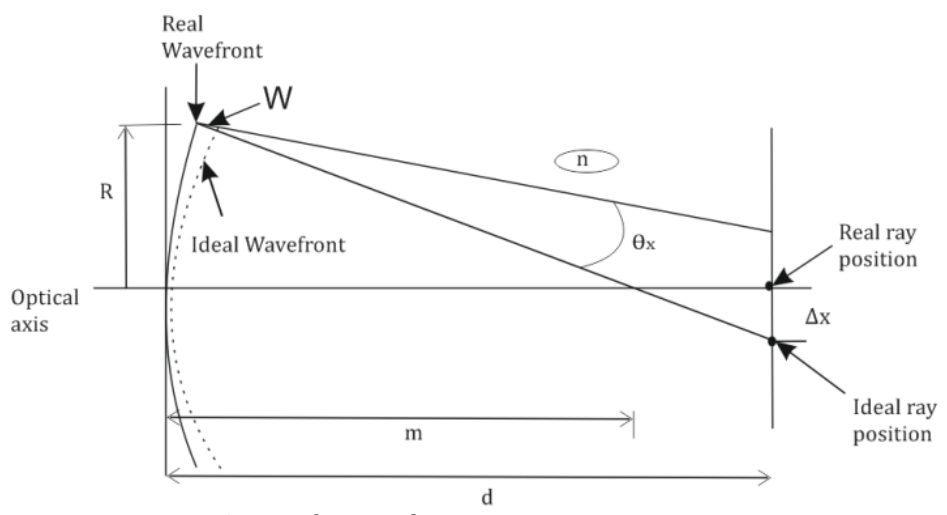

Fig.8.Wavefront and ray tracing in a Hartman test.

Applying the trapezoidal rule get the expression for surface's deviation at a $N$ th point from the position that could have a point if the wavefront was ideal. Ideal wavefront comes from a reference sphere. This technique offers several advantages such as that it is possible to remove the radial symmetry that the Placido technique presents and therefore the ambiguity caused by skew rays, since there is a point-to-point relationship between the screen and the image. Another advantage is the simplicity of the technique compared to other interferometric methods that have a lower dynamic range and require the control of many parameters during the test in addition to a large amount of data processing.

Mejía et al $[79,80]$ proposed a modification to the flat screen that is normally used in the Hartman test; they used an ellipsoidal screen with a distribution of 253 holes that contained a multimodal fiber optic segment, which they illuminated with a red fluorescent lamp. The illuminated ellipsoid produces a square arrangement of points $0.4 \mathrm{~mm}$ apart in $x, y$ directions. To generate the elevation maps and the curvature maps, they used the distortion of the Hartman pattern that corresponded to the surface under test in comparison with a reference sphere. Through an algorithm based on the Fourier Transform and a fitting with Zernike's polynomials, they obtained the surface's Sagitta and elevation maps.

The obtained results were reliable in comparison with a commercial topographer based on Placido rings, in measurements made to an individual. The comparison was qualitative because the measurements were not equivalent. They mentioned as a possible improvement to their system the use of higher order coefficients of Zernike polynomial to a better representation of irregularities in the corneal shape, in addition to increasing the resolution it is necessary to build more ellipsoids with greater density of points.

A variant of this test is known as Hartman-Shack [81]. Developed for defense purposes during the cold war by Roland Shack. Later was eventually used in the ophthalmological area for the first time by J. Bille et al [82] in the 1990s, to measure the corneal topography analyzing the wavefront reflected by the anterior surface and measure eye's aberration. The principle of operation consists in the generation of a point source in the retina. The light scattered by the retina that emerges from that source passes through the entire optical system of the eye forming a wavefront that contains the information of the ocular aberrations. This wavefront is sampled by an array of micro lenses (LA) as shown in Figure 9. Each lens focuses the portion of the wavefront at a point in the focal plane and is captured by a CCD camera; the PH diaphragm prevents from passing light reflected by the lens L3, while the lens L2 is used to align the eye with the axis of the optical system. The distance between the spots in its focal plane and the optical axis of the lenslet gives the local slope of the wavefront entering the lenslet. The distribution of the points that are captured in a CCD matrix allows the calculation of the shape of the whole wavefront. The aberrations induced by the eye can 
be described mathematically with the Zernike polynomials. This technique has several advantages like that can be implemented with a no coherent light source, besides that it is simple and versatile.

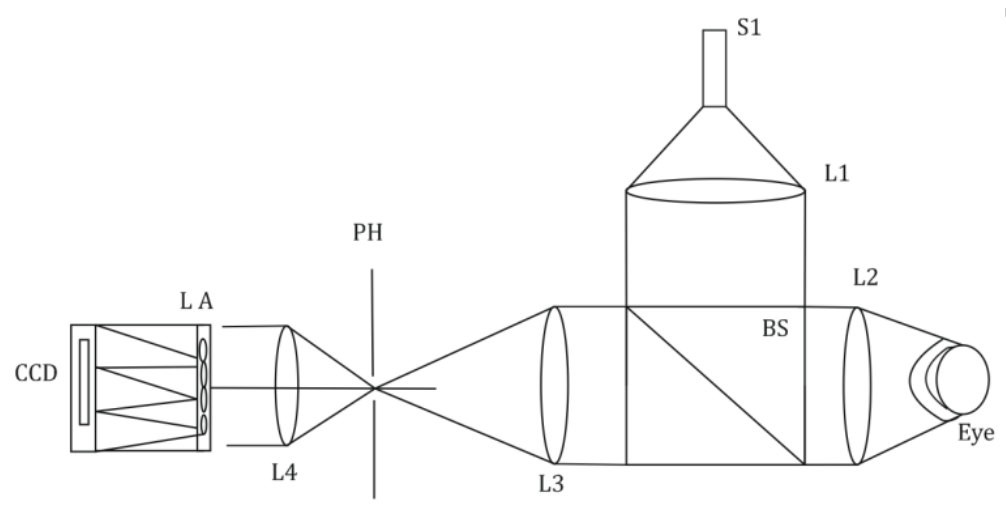

Fig.9.Basic optical set-up for Hartman-Shack test.

J. Yang et al. [83] implemented a system for corneal topography based on the Hartman-Shack sensor. The optical principle to measure the corneal surface is based on a lens focusing a beam on an ideal reference sphere, whose center of curvature is located exactly at the focus of the lens; because the anterior surface of the sphere is perfectly spherical, reflected rays follow the same optical path as the incident rays in the opposite direction. If the surface of the sphere is perfect, the reflected rays will follow a different optical path. In this way, the information of the differences in the elevation of the surface is recorded in the wavefront and with the Hartman-Shack sensor; the position of the points of the reference surface and the surface under test can be compared. Use of the modal method to reconstruct the front of the wave that is described in terms of functions of a few coefficients by multiplying the Zernike polynomials. The distribution of the wavefront slope was determined by comparing the measured centroids to a reference, by means of a mathematical model that relates the centroid position $(x, y)$ of the reference wavefront, those of the measured surface, the wavelength of the source and the distance between the lens set array and the detector. The measurements in a cylindrical eye model of $10 \mathrm{~mm}$ and $20 \mathrm{~mm}$ in diameter and height respectively, obtaining a deviation in the measurements of $0.0011 \mu \mathrm{m}$ RMS.

Mendoza-Villegas et al [84] proposed an alternative null test, which is fast and low cost due to the setup and mathematical principles applied. Is based on geometrical optics, specifically, the principle that forming rays of an image in a spherical mirror follow straight line. From the equations of the rays, that form the image and the point where the rays intersect, the radius of curvature of a calibration sphere is calculated. The proposed method was developed through Gauss's equation analysis obtaining the follow equation (8):

$$
r=\left(\frac{2 h^{\prime} l}{h-h^{\prime}}\right)
$$

where $r$ is the curvature radius, $h$ object height, $h^{\prime}$ image height, $l$ is the distance from the object to spherical surface.The experimental set up was formed by a flat screen monitor that projects a point that serves as an object, and using a beam splitter forms an image on the calibration sphere that is send to a CCD camera. They captured a sequence of images formed by different point's radius in continuum processes that ends when all the area of interest in the sphere was analyzed. They simulated a conicoid, an image formation in it with the calculated radii of curvature then applied a null test with the expected images, and those obtained with the CCD camera. The preliminary none compensate results showed a minimum error of $0.036 \mathrm{~mm}$ and a maximum of $0.668 \mathrm{~mm}$. The main advantages of this method are the simplicity, short analysis time, and accurate measurement resulting in a low-cost implementation.

\section{3. c. Polarimetry}

Yasumatsu et al [85] proposed the use of topographic sensors by time domain polarimetry with pulses in the order of Tera-Hertz and time-of-flight (TOF) images. In this technique an elliptical coherent pulse is directed to a sample, the direction of the polarization vector of the reflected electric field is detected by means of an electro-optical signal of a pulse $(f s)$ infrared laser that acted as a gate. A pulse that is reflected by a level of the sample closest to the surface will first reach the detector that one reflected by a level farther from the surface, because the optical path length is shorter. The state of polarization of the electric field of 
the pulse recorded in the detector will change $\Delta \gamma$ corresponding to the pulse delay on the surface of the sample. The continuous measurement of the polarization state by means of the scanning of the sample in the $x, y$ directions, and allows the measurement of the depth of the profiles on the surface of the sample. They implemented the technique for measuring the surface of a fused silica lens, obtaining a radius of the surface after an adjustment made to 100 measurements of $r=463 \mathrm{~mm}$ with an uncertainty of $\pm 3 \%$ that was consistent with the value reported in the manufacturer's commercial catalog $r=463 \mathrm{~mm}$. This technique offers several advantages such as being a non-contact and low photon energy test. However, it has to be investigated on surfaces with much smaller radii of curvature, such as the cornea, in order to consider the direction of the reflected Tera-hertz pulses in each point, in addition to reducing the time of image acquisition in order to be implemented in vivo human corneas.

\section{3. d. Intensity sensor}

S. Gruppeta et al [86] proposed a method to obtain corneal topography based on the reconstruction of the curvature of the wavefront. They measure changes in local intensity by beam propagation produced with an incoherent light source (halogen lamp); with a filter that transmits at $600 \pm 40 \mathrm{~nm}$ with an intensity of 400nW. A collector lens focuses light on a pinhole, and then a beam splitter 70:30 sent the beam to a photographic lens, which focuses light on the center of curvature of the cornea. The wavefront reflected by the interface between the air and the tear film contains the aberrations caused by the tear film with respect to a perfect sphere. The reflected beam was divided with a 50/50 beam splitter and two achromatic doublets $(\mathrm{f}=50 \mathrm{~mm})$ collimated the beams on the CCD cameras of $50 \times 50 \mu \mathrm{m}$ per pixel. The wavefront was recovered by solving an equation with the Poisson's equation and the Neumann boundary conditions. The first approximation of the signal was considered like the Laplacian of the wavefront and its estimation was obtained in the Fourier domain. Because the sensor signal is the wavefront Laplacian multiplied by the pupil function, the signal boundaries were considered with a Gershberg-type algorithm. With measurements on surfaces with known topographies they obtained an error in the configuration of $0.005 \mu \mathrm{m}$, with an accuracy of $0.08 \mu \mathrm{m}$, and a repeatability of $0.002 \mu \mathrm{m}$. They presented a map of radial curvature but do not make a comparison of its method with respect to other measurement systems.

\section{3. e. Hirshberg test}

S. Brodie [87] proposed a method based on the Hirschberg test that consist in the angular visualization of an eye with strabismus measuring the displacement of the first Purkinje image formed by the corneal reflex with a hand light. Starting from the assumption that the cornea is spherical, he proposes a relationship between reflection displacement and rotation angle of eye. He translates into an integral equation which differentiation conduce to a second-order differential equation that models the corneal topography in the terms of the Hirshberg test data, this proposal only reached the mathematical model and was never taken to an implementation in experimental arrangements.

\section{Diffuse reflection techniques}

\section{4.a. Rasterstereography}

This technique consists of a projector that displays a mesh of bright stripes with different widths depending on the characteristics of the specimen to be inspected. The optical axis of the projector is oriented horizontally such that the projection slide is approximately parallel to the test surface. The camera is placed at a certain convergence angle $\theta$, and will record the pattern that forms on the surface under test [88]. An arrangement used in this technique is shown in Figure 10.

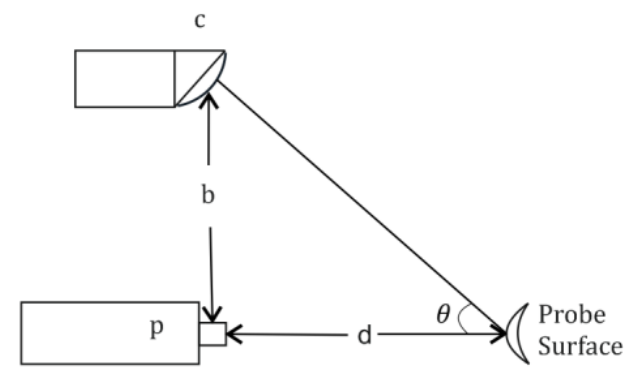

Fig.10. Basic rasterstereography system. 
This technique has been used for the measurement of the ocular surface [89]. Thanks to the geometry and from the triangulation method, the elevation of the cornea is measured comparing the horizontal displacement of the mesh pattern in the cornea and the same pattern of horizontal and vertical lines projected on a flat surface. Knowing the geometry of the pattern, the projection system and the optical path distances, is possible to calculate the $x, y, z$ coordinates of the surface. This technique has certain advantages such as, the ability to encompass the apical, peripheral and limbal zones of the cornea; furthermore, it does not depend on the reflectivity of the corneal surface. However, one disadvantage is that since cornea is not a diffusing surface, so fluorescent substances are used [90]. An accuracy of approximately 0.60 diopters has been reported for curvatures within $0.10 \mathrm{~mm}$ on metallic test surfaces [91].

A study by Belin et al [92] reports an accuracy of $0.1 D$ for ex vivo human eyes. Naufal et al [93] did a study with 100 healthy subjects using a system based on rasterstereography and concluded that this technique represents corneal topography with better accuracy than the commercial device based on Placido disks.

\section{4.b. Moiré Fringes}

The principle of operation of this technique consists in the projection of two grids of parallel lines, on a surface. The relief's surface can be obtained by means of the geometrical parameters of the configuration and the analysis of the grid from images captured with a digital camera. Considering a straight band that is projected from a point $A$ with a height $Z_{a}$ towards the point $C$ in the plane $z=0$. As you can see in Figure 11. If, that strip is observed from a point $B$ with a height $Z b$, this surface will intersect the strip in the plane $z=0$. Observing from point $\mathrm{B}$, the strip appears at point $E$, so that knowing the separation between points $E$ and $C$, the height of the object on the plane $z=0$ can be calculated. Assuming that both the observer and the projector of the fringes are placed at infinity and observer is located in a direction parallel to the z-axis in this case, equation 11 give the height of the object:

$$
f(x, y)+\frac{x}{\tan \theta}-\frac{s}{\sin \theta}=\frac{m d}{\sin \theta}
$$

where $\theta$ is the angle of inclination of the collimated light source; $m$ is the fringe number with $m=0$ located at the origin $(x=0)$; and $d$ is the period of fringes in the plane perpendicular to the illumination beam [94].

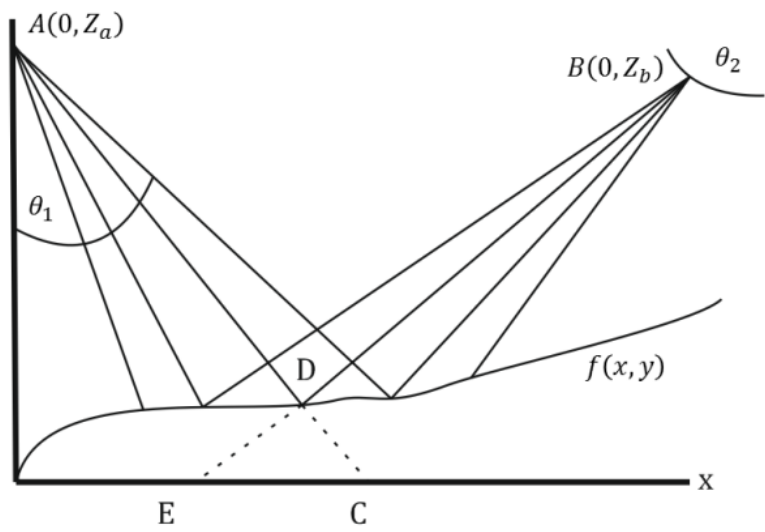

Fig.11. Periodic pattern projection on a surface [81].

Chang et al [95] used the projection of Moiré fringes with an experimental arrangement shown in Figure 12. They established the $z$ axis as the observation axis of camera $C$. A $473 \mathrm{~nm}$ collimated laser beam impinges on a grid $G 1$ with a period of $0.2822 \mathrm{~mm}$ and an angle of incidence $\alpha=30^{\circ}$, which is found in a motorized mount that moved at a speed $v=0.5644 \mathrm{~mm} / \mathrm{s}$ to induce a relative phase between grid $G 1$ and $G 2$. The phase was estimated using a least squares sinusoidal fitting algorithm. The fringes were deformed by the distribution of the height of the corneal surface and were captured with a CCD camera with a resolution of $1280 \times 1024$ pixels at 30 frames per second and an exposure time $\Delta t=0.033 \mathrm{~s}$ with a total capture time of $\Delta T=1 s$. Then he used a 2D phase unwrapping procedure for each pixel to reconstruct the profile. In a study with an ex vivo pig eye they obtained an error in the measurement of the height of approximately $3.5 \mu \mathrm{m}$, taking as reference the main vertical meridian of the corneal surface, which was established along the limit where the intensity of the light decreased substantially. A disadvantage of this technique is that a fluorescent substance is used to improve the visibility of the projected fringes. 


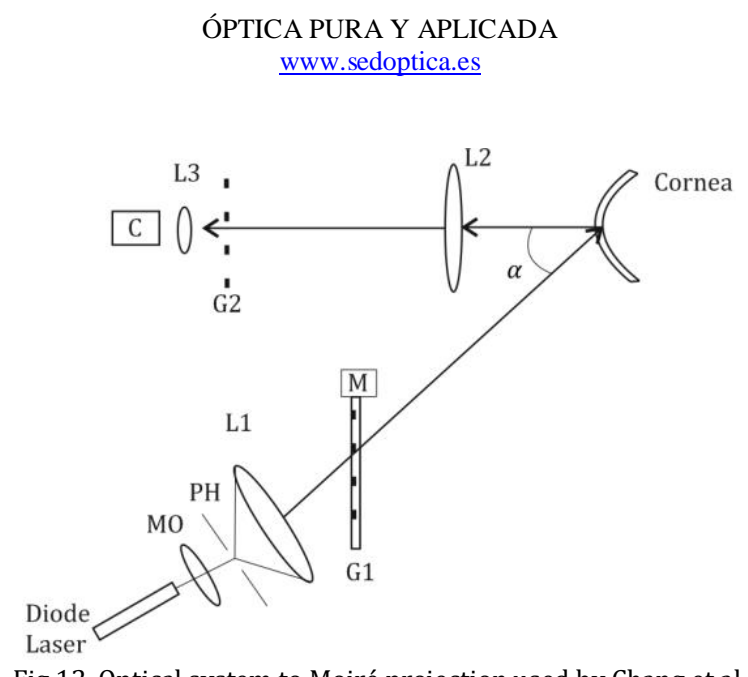

\section{4. c. Fourier transform profilometry}

Takeda [96] proposed Fourier transform Profilometry, and it has been used for the three-dimensional reconstruction of objects, and is one of the most important methods for the acquisition of three-dimensional images. This method is also based on the projection of a pattern of stripes on an object; the image with the distorted fringes on the object that is captured through a digital camera contains all the information of the three-dimensional profile of the object. According to Figure 13, there is a fictitious plane $R$, which serves as a reference to measure the height $h(x, y)$.

The pattern of fringes projected on the object and captured by the camera can be expressed by equation (10):

$$
g(x, y)=a(x, y)+b(x, y) * \cos \left[2 * \pi f_{0} x+\varphi(x, y)\right]
$$

where $g(x, y)$ is the intensity at the point $(x, y), a(x, y)$ is the retro-scattered light, $b(x, y)$ is the contrast between the fringes, $f_{0}$ is the fundamental frequency of the pattern of fringes and $\varphi(x, y)$ is the phase distortion between the values of $\pi$ to $-\pi$. The $x$ axis is the line that represents the plane $R$ in Figure 13 and the " $y$ " axis is perpendicular to the " $x$ " axis. For an object with a certain height variation described by the function $h(x, y)$, the pattern of captured fringes will be distorted and will contain the three-dimensional information of the object. Because the phase values are wrapped, means that if the angle formed by the vector (representing the complex number) with respect to the real axis is greater than $\pi / 2$, the calculation of the phase falls on the second branch of the tangent and arctangent function occurring a jump in the discontinuity. The calculation of the arctangent function does not distinguish between the second and the fourth quadrant or between the first and the third, being even more complicated if the complex vector has completed more than one cycle because not only the quadrant needs to be solved but also the branch in where the calculation of the phase falls. The solution to this problem is known as phase unwrapping and there are different techniques and algorithms to do it like the use of $z$ transform imaginary and real roots, Tribolet's Oppenheim's, Shafer's, Goldstein's, Flynn's algorithms among others.

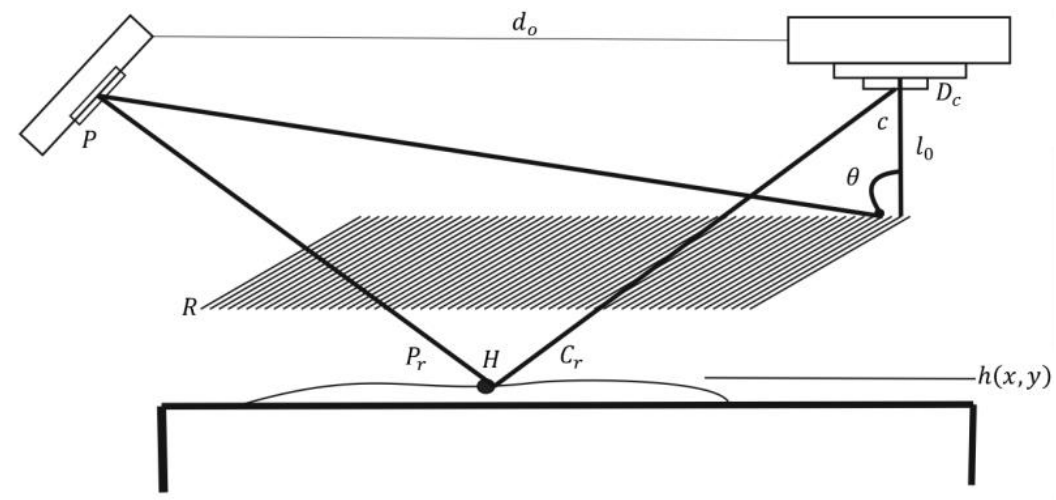

Fig.13. Basic set up of FTP system. 
After finding the unwrapped phase and considering the equivalence relation between the triangles $\triangle P H C$ and $\Delta P_{r} H C_{r}$, an expression (13) can be obtained for the height of the object as a function of the phase distribution [97]:

$$
h(x, y)=\frac{L \varphi_{z}(x, y)}{\varphi_{z}(x, y)-2 \pi f_{0} d}
$$

Profilometry has been used for the reconstruction of the topography of specular surfaces as convex lenses. Serrano et al [98] reported an error of $\pm 35 \mu \mathrm{m}$ in an area under study of $5 \mathrm{~cm}^{2}$ exceeding the error of $\pm 109 \mu \mathrm{m}$ obtained with the specular reflection technique similar to the Placido rings.

Recently R. Iskander et al [99] reported a study based on commercial ocular surface profilometer, which uses a double sequential projection architecture, which gives advantages over the substantial restrictions of the conventional Fourier transform profilometry. To achieve better accuracy the two projectors are located symmetrically with respect to the optical axis of the camera, with this design, this design provides a better robustness to the imprecision of the carrier frequency of other conventional configurations, because the angles between projectors are equal. In an essay with a group of 6 people, 18 measurements were made to each volunteer with the profilometer and a videokeratoscope of Placido for the purpose of making a comparative evaluation, which showed a difference of simulated keratometry between both instruments within the range of $\pm .5 \mathrm{D}$ for a $8 \mathrm{~mm}$ zone. The main disadvantage is that in the study they used a topical fluorescent solution as a guide for the correct acquisition of the images.

\section{Scattered Light}

\section{5. a. Slit-lamp System}

The Slit lamp is an instrument that consists of a high intensity light source in the form of a thin line with variable width by the user [100]. There are two types of slit lamps with and without a Galilean telescope as an amplification system [101]. The systems that obtain measurements of the anterior and posterior curvature, based on this technique, combine the projection of a slit lamp with the reflection principle used in the keratoscope. When a slit of light projects into the cornea, the light will reflect and refract due to the two surfaces (anterior and posterior). The light that is refracted will also be scattered by the tissue resulting in an image of a bright band being the outer and inner edge the front and rear surfaces respectively, which allows the measurement of the two surfaces Figure 14a. These optical properties are used to reconstruct the surface by means of the beam-ray triangulation method. The basic configuration of this technique is shown in Figure 14b. Some commercial equipment based on this technique uses two slit lamps projected at $45^{\circ}$ one to the left and one to the right of the axis of the camera with the eye, for approximately $1.5 \mathrm{~s}$ project 20 beams on each side, which allows the obtaining about 9000 points from the captured images [102].

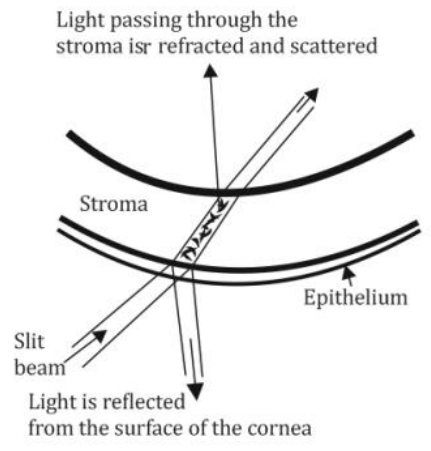

a)

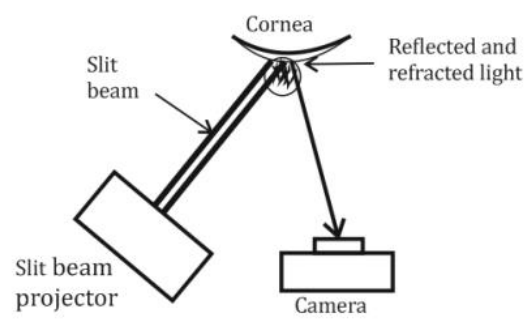

b)

Fig.14.a) Set-up pf a Slit-lamp system, b) optical phenomena that occurs in the cornea.

The information acquired is relative to other slit light on the surface and is described as the height on the $z$-axis compared to a reference plane. From the best fitting of sphere by least squares, you get an elevation map. One of the advantages of this technique is that in addition to the curvature and elevation maps, it is also capable of making pachymetry maps of the entire cornea. The main disadvantage of this technique is 
that the quality of the projected slit depends on the optical quality of the corneal surface. For example, in opaque, post-surgical or scarred corneas, there is an excessive scattering of the light resulting in difficulty to detect the edges causing a low accuracy than other techniques [103]. R. Martin [104], P. Lang [105] suggest that in a comparison of Placido rings, Scheimplufg image and slit lamp, they are not interchangeable within clinical practice and each equipment should be used in an independent manner, Scheimplufg image and slit lamp are mandatory in specialized practice, such as diagnosis and monitoring of pathologies and evaluation of refractive surgery.

\section{5. b. Scheimpflug imaging systems}

Conventional imaging application uses the object plane; the plane of the lenses and the image plane are parallel to each other. In the Scheimpflug imaging system the object plane, plane of the lenses and the image plane intersect in a line as shown in Figure 16. The main advantage of this geometry's system is that it achieves a great depth of focus. The principle of this system has been widely used in ophthalmology to obtain images of the entire anterior segment of the eye from the anterior surface of the cornea to the posterior surface of the lens. A variant Scheimpflug image system consists of two cameras; one located at the center for pupil's size detection and the other is mounted on a rotating rail that takes images at angles rom $0^{\circ}$ to $180^{\circ}$. This dual configuration measures the corneal thickness, in addition to other biometric data of the anterior segment that can be obtained and averaged to compensate the involuntary misalignment of the eye, in such a way that a correct value of the corresponding tested area is obtained. A commercial system of this technique is capable of taking 50 Scheimpflug images in less than two seconds. An image has 500 elevation points and each elevation map covers the entire anterior segment of the eye including the anterior and posterior surfaces, being able to generate 25,000 elevation points for each surface. The central thickness of the cornea can be calculated using equation 12 [106]:

$$
x=\frac{h}{\tan \alpha_{2}}-\frac{h}{\tan \alpha_{1}}+y
$$

where $x$ denotes corneal thickness, $h$ is a variable associated to the distance of the axis $O$ and the point $P$. In Figure 15, the green curve represents the anterior and posterior surface of the cornea, and the orange arc is the virtual image of the posterior surface. This technique can generate curvature and elevation maps and can be combined with the Placido technique to obtain a more accurate calculation of the central curvature of the anterior surface.

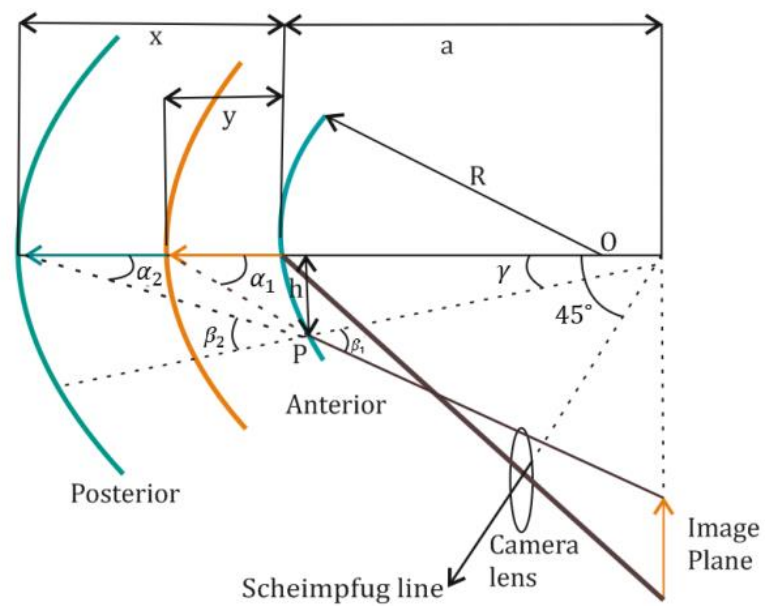

Fig.15.Scheimpflug image system with object, lens and image planes intersect in a line [93].

The main disadvantage of this technique is that Scheimpflug imaging systems require sophisticated calibration methods for their proper functioning [107]. But as a direct measurement is barely affected by misalignment of images out of center, being able to obtain deviations of $10 \mu \mathrm{m}$ in the measurement of corneal thickness for images out of center by $0.3 \mathrm{~mm}$.

Recent studies $[108,109]$ demonstrate the utility of this technique in the diagnosis and monitoring of keratoconus. In addition, through the modality of corneal tomography that this technique offers, monitoring 
the risk of ectasia [110,111]. Zhe Xu et al [112] reported the application of this technique with the Zernike polynomials for the subclinical detection of Keratoconus.

\section{Image formation}

\section{6. a. Image processing of conventional photography}

Recently, there has been an interest in the development of medical applications for smartphones; the reason is due to all advantages of using this kind of digital platforms like portability, accessibility and low cost. Ophthalmology has not been the exception; recently the use of mobile telephones for the detection of topographic pathologies has been reported.

A. Patel et al [113] proposed a method based on the Blinn-Phong scheme. With the camera of a cellphone, they captured a series of photographs moving the phone sharply, at the same time that the accelerometer and gyroscope recorded the position and orientation of the device in the moment that each photograph was taken. For each captured image, a model of an ideal cornea is generated from the perspective of the position and orientation of the collected data in each captured image, in such a way that each photo has its corresponding synthetic image. The data of the Blinn-Phong model depends on the material coefficients: environmental, diffuse and specular and is represented by the equation (13):

$$
c=(N \cdot L) \cdot d+(N \cdot H)^{\alpha} \cdot s+a
$$

where $a, b, s$, are the ambient, diffuse and specular colors respectively, $\alpha$ is the specular component that controls the brightness. The vector $N$ is the normal surface of the material, $L$ is the vector from the illumination source to a point on the surface and $H$ is the vector that falls between the normal vector and the illumination vector. The equation is applied to all the pixels of each image to obtain a new value in each pixel and thus generate the new image. The surfaces that were generated to represent the non-ideal forms of the eyes were phantom of a cone and a paraboloid. For each intensity distribution, curvature changes are apparently due to the geometry of each phantom, what opens the possibility of differentiating between various types of keratoconus. In this work only, the simulation of the method is presented, leaving the implementation in real images as future work.

B. Askarian et al. [114] they proposed a technique to obtain the curvature of the cornea, adopting the slope detection method from the images captured with the cellphone camera of an 8-megapixel and a resolution of $3264 \times 2448$. The analysis of the images consists of a preprocessing, which includes a cutout of the section of interest (cornea) conversion to a grayscale and $90^{\circ}$ image rotation. They identify the cornea's border using edge detection, and a morphological dilation that was developed to obtain a smoothed boundary. After preprocessing, a classification of keratoconus eyes from healthy eyes using the slope of the curvature of the cornea was implemented. The method they proposed was to detect the slope to measure cornea's curvature at different angles with $N$ different points. The algorithm classifies eyes based on each value of the slope sampled every $10^{\circ}$. In order to evaluate their method, they conducted a study with 10 participants (20 eyes) where six were diagnosed with keratoconus while 14 were healthy. The proposed method detects severe, advanced and moderate keratoconus with an accuracy of $93 \%, 86 \%, 67 \%$, respectively taken ophthalmologist's diagnoses as reference.

\section{6. b. Manual Keratometer}

The keratometer is an instrument used to measure radius of curvature " $r$ " of the cornea's anterior surface $[115,116]$. The basic principle of the keratometry is the image formation on the cornea when it is analyzed as a convex spherical mirror. Using the double prism procedure, the size of the reflected image is measured with which the value of " $r$ " is determined and converted to dioptric values using the ketarometric index of refraction $n_{k}=1.3375$. With this analysis, it is assumed that the image formed with respect to the object is virtual, right and reduced, located inside the eyeball. The equation (14) of the keratometer is the following [117]:

$$
r=\frac{2 d y}{h}
$$

where: $h$ is the height of the object, $d$ is distance between the object and image and $y$ is the height of the image. 
The power in diopters is given by equation 15 :

$$
\phi=\frac{337.5}{r}
$$

The keratometer only measures the central radius of curvature and assumes that the surface being measured is spherical which is not true because the cornea resembles more to a conical section. In addition, this instrument does not cover the total area of interest, limited to the central 3mm [26]. Another useful application of keratometer is for fitting contact lenses, it measure its base curve which is the radius of curvature of its back surface, often converted to diopters using the keratometer index of refraction [118].

\section{Conclusions}

Some pathologies of the eye that involve the anterior segment, along the multiple refractive errors of the cornea, require an accurate measurement of their dimensions and optical properties, for an adequate diagnosis and treatment. For this reason, there are different methods and techniques that have been developed and implemented during the last five decades. With the advancement of technology, some have been improved and new proposals have emerged, in order to obtain a safe, reliable and accurate measurement.

OCT is a commercially implemented technique that provides reliable pachymetric measurements, so that some authors suggest that it should be preferred over Scheimpflug imaging techniques for measurements of corneal thickness and the posterior surface of the eye. The principal challenge to get a correct shape representation from OCT is the problem of a not good fit to the data and the smoothness properties of the image.

In the case of the most recent interferometric methods, it is not necessary to reconstruct the corneal surface taking into account a reference sphere, due to its non-null nature. This technique is far more accurate than the classic Placido Rings, and can be considered the most commonly used, so it can be considered the most sensitive technique with a resolution in height of less than $5 \mu \mathrm{m}$. However, it has the disadvantage that its implementation is very sophisticated since it requires a controlled environment and a high computing capacity; as a result, the cost is considerably high.

Techniques based on specular reflection whose measurement principle is based on ray trace from the image that is formed by the reflection of a pattern on the cornea, have a disadvantage and is that sample's information points come from the tear film, so if there is any irregularity in this thin surface the measurement will be affected. But its great advantage is its low complexity for its implementation and in some cases the low cost.

The most common technique is the Placido rings which have several limitations like lack of complete coverage corneal area, non-pachymetric maps capability, and skew ray error. Even though is still used for its simplicity and full commercial availability, is not the best measurement practice due to its dependence on conditions for a correct shape representation.

Moiré deflectometry is a technique that can be used for the measurement of wavefront aberrations by establishing a relationship between the degree of distortion and the deflection angles, as well as for the radius of curvature. This technique can be as sensitive as interferometry. Despite this, the application of this technique for corneal topography has been a line of research with almost no development in recent years.

The phase measuring deflectometry was recently applied to corneal topography with promising results but needs to change in their set-up configuration to capture an align image to improve measurements.

The color led reflections is a technique that provides a trustworthy measurement comparable with other commercial technique like those based in slit-lamp systems.

Within specular reflection, the null Hartman test stands out as a reliable method of measurement. The method projects a spot pattern under the cornea and captures it with a detector, in order to compare it and obtain the transversal aberration. The major advantages are easy and low-cost implementation. Other null test configurations have been explored with promising results, but they need to overcome various limitations to offer a robust test that is viable for commercial implementation. 
The polarimetry by time of flight is a technique that needs to improve aspects like time of acquisitions and the dimensions of samples to measure in order to be viable for human corneal topography.

Intensity sensor is a viable alternative technique to make static corneal topography and dynamic measurements of tear film buts its implementations requires of complex algorithms.

Diffuse reflection methods need to place a fluorescent substance in the eye, which may represent discomfort to the user in clinical practice. All of these methods use a reference plane to measure cornea elevation, calculating the curvature by numerical methods or by fitting it to a function. Generally, a triangular configuration is used (cornea, projector, and detector).

Nowadays Fourier transform profilometry technique can be found in commercially available topographers and with results comparable to the equipment based on based on Placido topographers. On the other hand, rasterstereography is no longer commercially available although it was the first technique to offer elevation maps. The advantage of both systems is that they can measure the entire surface of the cornea.

Moiré fringe projection is a technique that offers some advantages as a high resolution, low sensitive to displacements along the optical axes and corneal coverage area that can be analyzed is comparable with other techniques like Placido disk.

The scattered techniques allow to measure other structures like anterior chamber depth and posterior corneal surface that cannot be measured by other kinds of techniques. Their principal limitations are that the cornea have to be ideal scattered properties to capture a correct image of both corneal surfaces.

The conventional photography with smartphones for obtaining corneal parameters it is a relatively new research area that right now is limited in accuracy and should be taken into account just for a preliminary diagnostic test and not for a determinant diagnostic study.

\section{Acknowledgements}

The authors wish to thank the financial support of Tecnológico Nacional de México and Consejo Nacional de Ciencia y Tecnología (México). 